\title{
Effective bounds from ineffective proofs in analysis: an application of functional interpretation and majorization*
}

\author{
Ulrich Kohlenbach \\ Fachbereich Mathematik, J.W.Goethe-Universitt \\ Robert-Mayer-Str. 6-10, 6000 Frankfurt am Main, FRG
}

\begin{abstract}
We show how to extract effective bounds $\Phi$ for $\forall u^{1} \forall v \leq_{\gamma} t u \exists w^{\eta} G_{0}$-sentences which depend on $u$ only (i.e. $\forall u \forall v \leq_{\gamma} t u \exists w \leq_{\eta} \Phi u G_{0}$ ) from arithmetical proofs which use analytical assumptions of the form $(*) \forall x^{\delta} \exists y \leq_{\rho} s x \forall z^{\tau} F_{0} \quad\left(\delta, \rho, \tau\right.$ are arbitrary finite types, $\eta \leq 2, G_{0}, F_{0}$ are quantifier-free and $s, t$ closed terms). If $\tau \leq 2,(*)$ can be weakened to

$\forall x^{\delta}, z^{\tau} \exists y \leq_{\rho} \quad s x \forall \tilde{z} \leq_{\tau} z F_{0}$. This is used to establish new conservation results about weak Knig's lemma WKL. Applications to proofs in classical analysis, especially uniqueness proofs in approximation theory, will be given in subsequent papers.
\end{abstract}

\section{Introduction and basic notions}

Various theorems in classical analysis have the form

$$
A \equiv \forall x \in X \exists y \in K_{x} \subseteq Y A_{1}(x, y)
$$

where $X, Y$ are complete separable metric spaces, $K_{x}$ is compact in $Y$ and $A_{1}$ is purely universal. If an analytical sentence $B$ is proved in using besides lemmata $A$ only arithmetical constructions and induction, then $A \rightarrow B$ is provable in classical arithmetic $\mathcal{A}$ (formulated in the language of all finite types). This is the case for an important class of uniqueness theorems (e.g. in approximation theory). Here $B$ is essentially of the form

$$
\forall u \in U, v \in V_{u} \exists k \in \mathbb{N} B_{1}(u, v, k),
$$

where $U, V$ are complete separable metric spaces, $V_{u}$ is compact in $V$ and $B_{1} \in \Sigma_{1}^{0}$.

Using a suitable standard representation of such spaces, $A$ and $B$ can be expressed in $\mathcal{A}$ as $\tilde{A} \equiv \forall x^{1} \exists y \leq_{1} s x \forall z^{0 / 1} A_{0}$ and $\tilde{B} \equiv \forall u^{1} \forall v \leq_{1} t u \exists k^{0} B_{0} \quad\left(A_{0}, B_{0}\right.$ are quantifier-free and $s, t$ closed terms).

In this paper we establish results which are (in their simplest form) of the following type:

¿From a proof of $(*) \tilde{A} \rightarrow \tilde{B}$ in $\mathcal{A}$ one can extract an effective bound $\Phi$ for $\exists k$ in $\tilde{B}$ which depends on $u$ only, i.e.

$$
\tilde{A} \rightarrow \forall u^{1} \forall v \leq_{1} t u \exists k \leq_{0} \Phi u B_{0} .
$$

This also holds if $x, y, z, v$ have arbitrary types and $k$ has a type $\eta \leq 2$.

\footnotetext{
${ }^{*}$ The results of this paper (except $2.9,2.12,2.15,3.8$ and 4.17 ) form the main part of chapter 7 of my dissertation Kohlenbach (1990). Some of them were presented at the 1989 European Summer Meeting of the ASL in Berlin. I am grateful to Prof. H. Luckhardt for many stimulating discussions and helpful suggestions.
} 
A similar extraction yields a bound $\Psi$ for $z$ in $\tilde{A}$ :

$$
\forall u^{1}\left(\forall x^{\delta} \exists y \leq_{\rho} s x \forall z \leq_{\tau} \Psi u A_{0} \rightarrow \forall v \leq_{\gamma} t u \exists k \leq_{\eta} \Phi u B_{0}\right) \quad(\tau, \eta \leq 2) ;
$$

thus a stronger conclusion follows from a weaker assumption. A proof of $\tilde{A}$ is not needed for the extraction of $\Phi, \Psi$. The correctness of $\Phi$ follows from the truth of (the weakened) $\tilde{A}$.

We extract $\Phi$ and $\Psi$ using a combination of functional interpretation and pointwise majorizability of primitive recursive functionals of finite type. If only elementary instances of induction occur in the proof of $(*)$ then $\Phi$ and $\Psi$ are simple constructions in the numerically relevant terms and datas of the proof.

As logical applications of these results we obtain new conservation results for weak Knig's lemma WKL, e.g. conservation w.r.t. $\forall u^{1} \forall v \leq_{\gamma} t u \exists w^{\eta} B_{0}$-sentences.

Mathematical applications to uniqueness proofs in approximation theory yielding new numerical estimates for rates of convergency will be given in subsequent papers.

Let $E-P A^{\omega}$ be the classical extensional arithmetic in all finite types (i.e. $\left(E-H A^{\omega}\right)^{c}$ in the notation of Troelstra (1973)), where the set $\tilde{T}$ of all finite types is given by the clauses

$0 \in \tilde{T}$ and $\rho, \tau \in \tilde{T} \Rightarrow \rho(\tau) \in \tilde{T}$ (each functional of type $\rho(\tau)$ maps objects of type $\tau$ to objects of type $\rho$; we often omit brackets which are uniquely determined and write e.g. $0(00)$ for $0(0(0)))$. If the axiom of extensionality for each type is replaced by a quantifier-free rule of extensionality

$$
E R-\text { qf } \frac{A_{0} \rightarrow s={ }_{\rho} t}{A_{0} \rightarrow r[s]={ }_{\tau} r[t]},
$$

where $A_{0}$ is quantifier-free, then one obtains the system $W E-P A^{\omega}$. For the corresponding theories with intuitionistic logic only, we write $E-H A^{\omega}$ and $W E-H A^{\omega}$. All these systems $\mathcal{T}$ have the same quantifier-free part qf $-\mathcal{T}$ (in the sense of Troelstra (1973),1.6.13) which we call T. $T$ is an extensional version of the Hilbert (1926)/Gdel (1958) calculus of primitive recursive functionals of finite type.

For functionals $x_{1}^{\rho}, x_{2}^{\rho}$ we have the following natural inequality relation:

$$
\left\{\begin{array}{l}
x_{1} \leq_{0} \quad x_{2}: \equiv x_{1} \leq x_{2} \quad(\text { where " } \leq " \text { is primitive recursively defined as usual) } \\
x_{1} \leq_{\tau \delta} \quad x_{2}: \equiv \forall y^{\delta}\left(x_{1} y \leq_{\tau} x_{2} y\right) .
\end{array}\right.
$$

The axiom schema of full choice is defined by $A C:=\bigcup_{\rho, \tau \in \tilde{T}}\left\{(A C)^{\rho, \tau}\right\}$, where

$(A C)^{\rho, \tau}: \forall x^{\rho} \exists y^{\tau} A(x, y) \rightarrow \exists Y^{\tau \rho} \forall x^{\rho} A(x, Y x)$. 
Quantifier-free choice $A C$-qf is $A C$ restricted to quantifier-free formulas.

We begin our investigations in this paper with the following observation:

Let $A \in \mathcal{L}\left(W E-P A^{\omega}\right)$ be a closed formula (i.e. a sentence) having the form

(1) $\forall x^{1} \forall \tilde{x} \leq_{\rho} s x \exists y^{\tau} A_{0}(x, \tilde{x}, y)$

with $A_{0}$ quantifier-free and $s^{\rho 1} \in T$ closed, where $\rho \in \tilde{T}$ is arbitrary and $\tau \leq 2$ (i.e. $\tau=0,00$ or $0(00))$. If $A$ is provable in $W E-P A^{\omega}+A C$-qf then by a combination of functional interpretation and a pointwise version of hereditary majorization of functionals from $T$, one can extract a closed term $\Phi^{\tau 1} \in T$ from any given proof such that

(2) $W E-H A^{\omega} \vdash \forall x \forall \tilde{x} \leq s x \exists y \leq_{\tau} \Phi x A_{0}(x, \tilde{x}, y)$

(see Kohlenbach (1992),3.4 and the proof of 2.3 below). This allows the extraction of uniform bounds $\in T$ for sentences of the form (1) which are proved in $W E-P A^{\omega}+A C$-qf from assumptions having the form (3) $\forall x^{\delta} \exists y \leq_{\rho} s x \forall z^{\eta} A_{0}$ :

(4) $W E-P A^{\omega}+A C-\mathrm{qf} \vdash \forall x^{\delta} \exists y \leq_{\rho} s x \forall z^{\eta} A_{0} \rightarrow \forall u^{1} \forall v \leq_{\gamma} t u \exists w^{\tau} B_{0} \quad$ implies

$W E-P A^{\omega}+A C-\mathrm{qf} \vdash \forall Y \leq_{\rho \delta} s \forall u \forall v \leq t u \exists x, z, w\left(A_{0}(x, Y x, z) \rightarrow B_{0}\right)$.

Using the extraction of the bound for " $\exists w$ " above, one can construct a closed term $\Phi \in T$ such that

$$
W E-H A^{\omega} \vdash \exists Y \leq s \forall x, z A_{0}(x, Y x, z) \rightarrow \forall u \forall v \leq t u \exists w \leq_{\tau} \Phi u B_{0}
$$

and therefore

(5) $W E-H A^{\omega}+A C \vdash \forall x \exists y \leq s x \forall z A_{0} \rightarrow \forall u \forall v \leq t u \exists w \leq_{\tau} \Phi u B_{0}$ (2.3).

For $\tau=0$ this yields an algorithm $\tilde{\Phi} \in T$ for $w$ :

$$
\tilde{\Phi} u v=\left\{\begin{array}{l}
\min w \leq_{0} \Phi u\left[B_{0}(u, v, w)\right] \text { if such a } w \text { exists } \\
0^{0} \text { otherwise }(2.5)
\end{array}\right.
$$

Using a more complicated extraction (2.9) one can prove (5) within $E-H A^{\omega}+A C$-qf, thus avoiding the axiom of choice for formulas containing quantifiers.

The result (5) is of mathematical interest since various important (non-constructive) theorems of classical analysis have the logical form

(6) $\forall x^{1} \exists y \leq_{1} s x \forall z^{0 / 1} A_{0}$ (modulo a suitable standard representation of complete separable metric spaces and compact metric spaces) and are therefore admissible premises for our extraction (5). In 
the case where only premises of the form (6) are used, (5) can be proved even in $W E-H A^{\omega}$ (thus choice can be avoided altogether) and if furthermore $\gamma \leq 1$, then $W E-P A^{\omega}$ may be replaced by $E-P A^{\omega}$ in (4) (see 3 below).

Examples of theorems of analysis having the logical form (6) are:

a) $\forall f \in \mathcal{C}[0,1] \exists x_{0} \in[0,1]\left(f\left(x_{0}\right)=\sup _{x \in[0,1]} f(x)\right)$,

b) The intermediate value theorem for $f \in \mathcal{C}[0,1]$ and the mean value theorem for the Riemann integral.

A more specific example is

c) the existence of a best approximation and an extremal alternant for the best Chebycheff approximation of $f \in \mathcal{C}[0,1]$ by algebraic polynomials of degree $\leq n\left(P_{n}\right)$ :

(7) $\forall f \in \mathcal{C}[0,1] \exists p_{b} \in P_{n},\left(x_{1}, \ldots, x_{n+2}\right) \in[0,1]^{n+2}, j \in\{0,1\}\left(\left\|f-p_{b}\right\|_{\infty}=\operatorname{dist}\left(f, P_{n}\right)\right.$

$$
\left.\wedge \bigwedge_{i=1}^{n+1}\left(x_{i+1}-x_{i} \geq 0\right)^{1} \wedge \bigwedge_{i=1}^{n+2}(-1)^{i+j}\left(p_{b}\left(x_{i}\right)-f\left(x_{i}\right)\right)=\operatorname{dist}\left(f, P_{n}\right)\right)
$$

where $\|\cdot\|_{\infty}$ denotes the sup norm and $\operatorname{dist}\left(f, P_{n}\right):=\inf _{p \in P_{n}}\|f-p\|_{\infty}$.

(Since $\left\|p_{b}-f\right\|_{\infty}=\operatorname{dist}\left(f, P_{n}\right)\left(\leq\|f\|_{\infty}\right) \Rightarrow\left\|p_{b}\right\|_{\infty} \leq 2\|f\|_{\infty}, P_{n}$ can be replaced by

$K_{f, n}:=\left\{p \in P_{n}:\|p\|_{\infty} \leq 2\|f\|_{\infty}\right\}$ which is compact and therefore has a bounded standard representation).

Such sentences are in fact used in proofs of $\forall u^{1} \forall v \leq_{1} t u \exists w^{0} B_{0}$-theorems in classical analysis. Examples are uniqueness proofs, e.g. proofs of the uniqueness of the best Chebycheff approximation. Uniqueness in this case means

$$
(8) \forall f \in \mathcal{C}[0,1], n \in \mathbb{N}, p_{1}, p_{2} \in K_{f, n}\left(\left\|p_{1}-f\right\|_{\infty}=\operatorname{dist}\left(f, P_{n}\right)=\left\|p_{2}-f\right\|_{\infty} \rightarrow\left\|p_{1}-P_{2}\right\|_{\infty}=0\right) .
$$

If we now write " $\forall l^{0}\left(\left\|p_{i}-f\right\|_{\infty}-\operatorname{dist}\left(f, P_{n}\right) \leq \frac{1}{l+1}\right) "$ for $"\left\|p_{i}-f\right\|_{\infty}=\operatorname{dist}\left(f, P_{n}\right)$ " and " $\forall k^{0}\left(\left\|p_{1}-p_{2}\right\|_{\infty}<\frac{1}{k+1}\right)$ " for " $\left\|p_{1}-p_{2}\right\|_{\infty}=0$ " then (8) transforms (modulo standard representa-

\footnotetext{
${ }^{1}$ If $\operatorname{dist}\left(f, P_{n}\right)>0$ (i.e.f $\left.\notin P_{n}\right)$ then the last conjunction implies $x_{i+1}-x_{i} \geq 0 \rightarrow x_{i+1}>x_{i}$.
} 
tions of $\mathcal{C}[0,1]$ and $\left.K_{f, n}\right)$ into

$$
(9) \forall f^{1}, n^{0} \forall p_{1}, p_{2} \leq_{1} s(f, n) \forall k^{0} \exists l^{0}(\underbrace{\left\|p_{1 / 2}-f\right\|_{\infty}-\operatorname{dist}\left(f, P_{n}\right) \leq \frac{1}{l+1} \rightarrow\left\|p_{1}-p_{2}\right\|_{\infty}<\frac{1}{k+1}}_{F: \equiv}) .
$$

Since $\operatorname{dist}\left(f, P_{n}\right)$ and $\|\cdot\|_{\infty}$ are primitive recursively computable (we consider $f \in \mathcal{C}[0,1]$ always endowed with a modulus of uniform continuity) the premise part of $\mathrm{F}$ is (equivalent to) a $\forall^{0}$-formula, while the conclusion is a $\exists^{0}$-formula. Therefore, by prenexing, $\mathrm{F}$ has the form $\exists^{0} F_{0}$. Hence (9) is of the form (1). Furthermore (9) can be proved from assumptions of the form (6), for example a) and c), relative to $W E-P A^{\omega}+A C^{0,0}-\mathrm{qf}$. Therefore our logical analysis of such uniqueness proofs yields a realization $\Phi f n k$ of $l$ which does not depend on $p_{1}, p_{2}$ :

$$
(10) \forall f, n ; p_{1}, p_{2} \leq s(f, n), k\left(\left\|p_{1 / 2}-f\right\|_{\infty}-\operatorname{dist}\left(f, P_{n}\right) \leq \frac{1}{\Phi f n k+1} \rightarrow\left\|p_{1}-p_{2}\right\|_{\infty}<\frac{1}{k+1}\right) .
$$

For a best approximant $p_{b}^{f, n} \in P_{n}$ of $f$ this yields

$$
(11) \forall f, n ; p \leq s(f, n), k\left(\|p-f\|_{\infty}-\operatorname{dist}\left(f, P_{n}\right) \leq \frac{1}{\Phi f n k+1} \rightarrow\left\|p-p_{b}^{f, n}\right\|_{\infty}<\frac{1}{k+1}\right) .
$$

A classical result in approximation theory says (non-constructively) that a best approximant $p_{b}^{f, n}$ always exists. This can be constructivized to

$$
\forall f \in \mathcal{C}[0,1], n, l \bigvee_{\text {eff. }} p_{l} \in K_{f, n}\left(\left\|p_{l}-f\right\|_{\infty}-\operatorname{dist}\left(f, P_{n}\right)<\frac{1}{l+1}\right)
$$

Now let $\Psi(f, n, l)$ denote an algorithm for $p_{l}$ then (11) implies

$$
(12) \forall f \in \mathcal{C}[0,1], n, k\left(\left\|\Psi(f, n, \Phi f n k)-p_{b}^{f, n}\right\|_{\infty}<\frac{1}{k+1} \wedge \Psi(\ldots) \in K_{f, n}\right), \text { i.e. }
$$

$\lambda k . \Phi f n k$ is a modulus of convergence for the sequence $(\Psi f n l)_{l \in \mathbb{N}} \stackrel{l \rightarrow \infty}{\longrightarrow} p_{b}^{f, n}$ (w.r.t. the norm $\left.\|\cdot\|_{\infty}\right)$. One easily shows that $\lambda k .2 \Phi f n k+1$ is also a modulus of (pointwise) continuity of the Chebycheff projection

$$
\begin{gathered}
\mathcal{P}: \mathcal{C}[0,1] \times \mathbb{N} \rightarrow \bigcup_{n} P_{n} \\
(f, n) \mapsto p_{b}^{f, n} .
\end{gathered}
$$

All this will be elaborated in subsequent papers where we analyse, in particular, various classical proofs for the uniqueness of best Chebycheff approximation and extract the corresponding moduli $\Phi$ with all numerical details. This yields new a priori moduli of uniqueness and continuity and estimates for strong unicity and Lipschitz continuity (which improve results of D. Bridges $(1980,1982)$ by an $\mathrm{n}$ (=degree) in the exponent).

In this paper we develope the underlying proof-theoretic method and apply it to obtain conservation results for weak Knig's lemma $W K L$ (4.1). 
By using an argument analogous to the one for (5) one can construct a bound $\Psi \in T$ for " $\forall z$ " if $\eta \leq 2$ such that

(13) $W E-H A^{\omega}+A C \vdash \forall u\left(\forall x \exists y \leq s x \forall z \leq_{\eta} \Psi u A_{0} \rightarrow \forall v \leq t u \exists w \leq_{\tau} \Phi u B_{0}\right)(2.13)$.

For $\eta=0$, this reduces the logical complexity of the implicative assumption

$$
\forall x \exists y \leq s x \forall z^{0} A_{0}
$$

to

$$
\forall x, z \exists y \leq s x \bigwedge_{k=0}^{z} A_{0}(x, y, k)
$$

where $\bigwedge_{k=0}^{z} A_{0}(x, y, k)$ can be expressed in a quantifier-free way in $W E-H A^{\omega}$.

In particular, proofs using sentences as e.g.

$$
\forall f \in \mathcal{C}[0,1] \exists x_{0} \in[0,1]\left(f x_{0}=\sup _{x \in[0,1]} f x\right)
$$

can be transformed into proofs which use only their " $\varepsilon$-versions"

$$
\forall f \in \mathcal{C}[0,1], k \in \mathbb{N} \exists x_{0} \in[0,1]\left(\left|f x_{0}-\sup _{x \in[0,1]} f x\right| \leq \frac{1}{k+1}\right) .
$$

These $\varepsilon$-versions are usually provable in $W E-H A^{\omega}$ (Thus e.g. the results for best approximation, mentioned above, can be verified within $\left.W E-H A^{\omega}\right)$.

Furthermore (13) can be generalized classically to formulas

$$
\begin{aligned}
& \forall u^{1} \exists a^{\delta} \forall b \leq_{\rho} r u a \exists w^{\tau} B_{0} \quad(\delta, \tau \leq 2) \\
& \text { - instead of } \forall u^{1} \forall v \leq t u \exists w B_{0} \quad \text { - yielding bounds (depending on } u \text { only) for } w \text { and } a \text { (2.12). }
\end{aligned}
$$

All the above results also hold for the restricted (in the sense of Feferman (1977)) systems $(W) E \widehat{-P} A^{\omega} \wedge,(W) E \widehat{-H}$ and $\widehat{P R}$ instead of $(W) E-P A^{\omega},(W) E-H A^{\omega}$ and $T$ with quantifier-free induction and elementary recursor constants only.

Finally we show that $W K L$ is an admissible premise (6) since

$$
W \widehat{E-} H A^{\omega} \wedge \vdash W K L \leftrightarrow \forall x^{1} \exists y \leq_{1} \lambda k \cdot 1 \forall z^{0} A_{0}^{K},
$$

for a suitable quantifier-free formula $A_{0}^{K} \in \mathcal{L}\left(W \widehat{E-H} A^{\omega} \uparrow\right)$ and

$$
W \widehat{E-} H A^{\omega} \wedge \vdash \forall x, z^{0} \exists y \leq \lambda k .1 \bigwedge_{i=0}^{z} A_{0}^{K}(x, y, i)
$$


As a corollary we see that $W E-P A^{\omega}$ plus $A C$-qf and $W K L$ is a conservative extension of $W E-H A^{\omega}\left(W E-P A^{\omega}+A C-\mathrm{qf}\right)$ w.r.t. sentences of the form $\forall u^{1} \forall v \leq_{\gamma} t u \exists w^{\tau} A_{0} \quad\left(\forall u^{1} \forall v \leq_{\gamma} t u \exists w^{0} \forall z^{1} A_{0}\right)$ where $\gamma, \tau \in \tilde{T}$ are arbitrary.

By constructing a counterexample we show that $W K L$ is not conservative for $\forall x^{2} \exists y^{1} A_{0}(x, y)$ sentences (4.11).

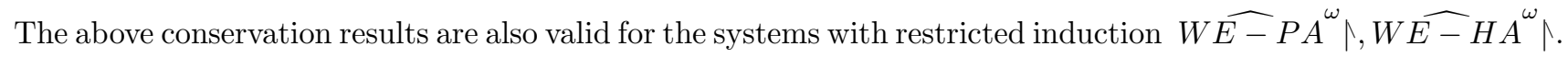

If $\gamma, \tau \leq 1$ then $W E-P A^{\omega}\left(W \widehat{E-P} A^{\omega} \wedge\right)$ can be replaced by $E-P A^{\omega}\left(\widehat{E-P} A^{\omega} \wedge\right)$.

The following conservation results can be found in the literature:

Sieg (1985) showed proof-theoretically that for the restriction $R C A_{0}$ of $\widehat{W E-P A^{\omega}} \uparrow+A C^{0,0}-\mathrm{qf}$ to objects of type 0,1 only $R C A_{0}+W K L$ is conservative over primitive recursive arithmetic PRA for $\Pi_{2}^{0}$-sentences. He used normalization of proofs and majorization of primitive recursive terms $t^{0}[f]$ with function parameters (primitive recursive in the sense of Kleene (1952)) to establish his results. H. Friedman previously proved the same result using modeltheoretic methods due to Kirby/Paris (1977). Feferman (1988) states the result also for $(W E-) \widehat{P A}^{\omega} \wedge+A C$-qf and refers to unpublished work of Sieg and himself for a (proof-theoretical) proof.

In Sieg (1987),(1991) a proof that $R C A_{0}+W K L$ is $\Pi_{1}^{1}$-conservative over $R C A_{0}$ is formulated as well as for the corresponding theory with full induction and various intermediate systems (with $\exists_{n}$-induction in Sieg's terminology): The proof relies on normalization of infinitary proofs using infinite terms (in the sense of Tait (1965)), but makes incorrect use of Herbrand normal forms in order to generalize $\forall f^{1} \exists n^{0} A_{0}$-conservation ( $A_{0}$ quantifier-free) to arbitrary $\Pi_{1}^{1}$-sentences and thus establishes conservation only for the former case which is a special form of our $\forall u^{1} \forall v \leq_{\gamma} t u \exists w^{\tau} A_{0^{-}}$ sentences (For details see Kohlenbach (1992 A) where a counterexample to Sieg's use of Herbrand normal form is given). There is mention in the literature, e.g. Sieg (1985), of an unpublished model-theoretic proof by Harrington that $R C A_{0}+W K L$ is $\Pi_{1}^{1}$-conservative over $R C A_{0}$ (more precisely for a variant of $R C A_{0}$ with set variables instead of function variables). This is generalized in Clote/Hajek/Paris (1990) to systems with $\Sigma_{n}^{0}$-induction (instead of $\Sigma_{1}^{0}$-induction, which is provable in $R C A_{0}$ and $\left.W \widehat{E-P} A^{\omega} \wedge+A C^{0,0}-\mathrm{qf}\right)$.

Finally there is the classical result (due to Kreisel (1963),(1966), Scott (1962) and Troelstra (1974)) that $W E-P A^{\omega}+W K L+A C-\mathrm{qf}$ is conservative over $P A$.

Furthermore we prove that, relative to $W E-P A^{\omega}+W K L$, each $\forall x^{1} \exists y \leq_{1} s x \forall z^{0 / 1} A_{0}$-sentence is equivalent to a $\forall n^{0} B_{0}$-sentence, where $A_{0}, B_{0} \in \mathcal{L}\left(W E-P A^{\omega}\right)$ are quantifier-free. An analogous result holds for $W \widehat{E-P} A^{\omega} \uparrow$ instead of $W E-P A^{\omega}$ (4.15). 
We conclude this paper by showing how one can extract bounds from proofs which use assumptions of the form $(*) \forall x^{1}\left(\forall w^{0} A_{0} \rightarrow \exists y \leq_{1} s x \forall z^{0} B_{0}(x, y, z)\right)$ (4.17). In this case, a proof of the $\varepsilon$-version of $(*)$ is needed (This contrasts to the assumptions $\forall x^{\delta} \exists y \leq_{\rho} s x \forall z^{\tau} A_{0}$ considered above).

\subsection{Notation}

The theories $E-P A^{\omega}, E-H A^{\omega}, W E-P A^{\omega}, W E-H A^{\omega}$ and $T$ all contain recursor constants $R_{\rho}$ with the defining axioms

$$
\left\{\begin{array}{l}
R_{\rho} 0 y z={ }_{\rho} y \\
R_{\rho}\left(S x^{0}\right) y z={ }_{\rho} z\left(R_{\rho} x y z\right) x,
\end{array}\right.
$$

where $y$ and $z$ are of type $\rho$ and $\rho 0 \rho$.

$s={ }_{\rho} t$ (for $\left.\rho=0 \rho_{k} \ldots \rho_{1}\right)$ is used as an abbreviation for $\forall y_{1}^{\rho_{1}}, \ldots, y_{k}^{\rho_{k}}\left(s y_{1} \ldots y_{k}=_{0} t y_{1} \ldots y_{k}\right)$ (resp. for $s y_{1} \ldots y_{k}={ }_{0} t y_{1} \ldots y_{k}$ in the quantifier-free calculus $T$ ) with different variables $y_{1}, \ldots, y_{k}$ not occuring in $s$ and $t$.

We often denote finite tuples $y_{1}, \ldots, y_{k}$ of functionals by $\underline{y}$.

If the constants $R_{\rho}$ are replaced by elementary recursor constants $\widehat{R}_{\rho}$ characterized by

$$
\left\{\begin{array}{l}
\widehat{R}_{\rho} 0 y z \underline{v}={ }_{0} y \underline{v} \\
\widehat{R}_{\rho}\left(S x^{0}\right) y z \underline{v}={ }_{0} z\left(\widehat{R}_{\rho} x y z \underline{v}\right) x \underline{v},
\end{array}\right.
$$

where $\underline{v}=v_{1}^{\rho_{1}} \ldots v_{k}^{\rho_{k}}$ such that $y \underline{v}$ is of type 0 , and if the schema of full induction is replaced by the axiom of quantifier-free induction

$$
(I A)-\mathrm{qf}: \forall f^{1}(f 0=0 \wedge \forall x(f x=0 \rightarrow f(S x)=0) \longrightarrow \forall x(f x=0)),
$$

then one obtains the restricted systems $\widehat{E-P} A^{\omega} \uparrow, \ldots$ ect. with quantifier-free part $\widehat{P R}$ due to Feferman (1977) (The functionals of $\widehat{P R}$ are essentially the primitive recursive functionals in the sense of Kleene (1959)).

All the systems above allow the definition of a term $\lambda x^{\tau} \cdot t^{\rho}[x] \in T(\widehat{P R})$ for each term $t^{\rho} \in T(\widehat{P R})$ such that $(\lambda x . t[x])\left(t^{\prime \tau}\right)={ }_{\rho} t\left[t^{\prime}\right]$ (see Troelstra (1973),1.6.8,1.8.4). For a theory $\mathcal{T}$ the language of $\mathcal{T}$ is denoted by $\mathcal{L}(\mathcal{T})$.

We usually use " $\forall x \leq_{\rho} y A ", " \exists x \leq_{\rho} y A ", " \bigwedge_{i=0}^{k} A(i) ", " \bigvee_{i=0}^{k} A(i)$ " as abbrevations for " $\forall x\left(x \leq_{\rho} y \rightarrow A\right)$ ", " $\exists x\left(x \leq_{\rho} y \wedge A\right) ", " \forall i \leq k A(i) ", " \exists i \leq k A(i)$ ". Furthermore " $\forall x ; \tilde{x} \leq_{\rho} s x A$ " stands for " $\forall x \forall \tilde{x} \leq_{\rho} s x A$ ", but " $\forall x, \tilde{x} \leq_{\rho} y A$ " stands for " $\forall x \leq_{\rho} y \forall \tilde{x} \leq_{\rho} y A$ ".

$A_{0}, B_{0}, C_{0}, \ldots$ denote quantifier-free formulas. 
By the principle of bounded choice we mean the schema

$$
(b-A C)^{\rho, \tau}: \forall Z^{\tau \rho}\left(\forall x^{\rho} \exists y \leq_{\tau} Z x A(x, y, Z) \rightarrow \exists Y \leq_{\tau \rho} Z \forall x A(x, Y x, Z) .\right.
$$

$(b-A C)^{\rho, \tau}$-qf $\left((b-A C)^{\rho, \tau}-\forall,(b-A C)^{\rho, \tau}-\forall^{b}\right)$ is $(b-A C)^{\rho, \tau}$ restricted to quantifier-free formulas (formulas having the form $\forall u^{\delta} A_{0}$ resp. $\left.\forall u \leq{ }_{\delta} v A_{0}\right) . b-A C:=\bigcup_{\rho, \tau \in \tilde{T}}\left\{(b-A C)^{\rho, \tau}\right\}$.

$(C)^{\rho}: \exists y^{0 \rho} \forall x^{\rho}\left(y x={ }_{0} 0 \leftrightarrow A(x)\right) \quad$ (comprehension),

$(M P)^{\omega}: \forall \underline{x}(A(\underline{x}) \vee \neg A(\underline{x})) \wedge \neg \neg \exists \underline{x} A(\underline{x}) \rightarrow \exists \underline{x} A(\underline{x}) \quad$ (Markov principle),

$\Sigma_{1}^{0}-A C^{0,0}: \forall x^{0} \exists y^{0}, z^{0} A_{0}(x, y, z) \rightarrow \exists f \forall x \exists z A_{0}(x, f x, z)$,

$\Sigma_{1}^{0}-I A: \forall f^{1}\left(\exists y^{0}(f 0 y=0) \wedge \forall x^{0}\left(\exists y(f x y=0) \rightarrow \exists y\left(f x^{\prime} y=0\right)\right) \rightarrow \forall x \exists y(f x y=0)\right),{ }^{2}$

$\Delta_{1}^{0}-C A: \forall x^{0}\left(\exists y^{0} A_{0}(x, y) \leftrightarrow \forall y^{0} B_{0}(x, y)\right) \rightarrow \exists f^{1} \forall x\left(f x=0 \leftrightarrow \exists y A_{0}(x, y)\right)$.

For a set $\Gamma$ of sentences $\in \mathcal{L}\left(W E-P A^{\omega}\right), W E-P A^{\omega} \oplus \Gamma$ means that the sentences from $\Gamma$ are added as new axioms to $W E-P A^{\omega}$ but that application of the extensionality rule is allowed only when $A_{0} \rightarrow s=t$ is proved in $W E-P A^{\omega}$ (i.e. without using the axioms $\Gamma$ ). $W E-P A^{\omega}$ satisfies the deduction theorem w.r.t. $\oplus$ but not w.r.t. + .

\subsection{Definition}

$\min _{\rho}\left(x_{1}^{\rho}, x_{2}^{\rho}\right)$ is defined by induction on the type $\rho$ :

$$
\left\{\begin{array}{l}
\min _{0}\left(x_{1}, x_{2}\right):=\min \left(x_{1}, x_{2}\right) \\
\min _{\tau \rho}\left(x_{1}, x_{2}\right):=\lambda y^{\rho} \cdot \min _{\tau}\left(x_{1} y, x_{2} y\right)
\end{array}\right.
$$

Clearly $\min _{\rho} \in \widehat{P R} \subset T$.

\subsection{Proposition}

1) $W \widehat{E-} P A^{\omega} \uparrow+A C^{0,0}-\mathrm{qf} \vdash \Sigma_{1}^{0}-A C^{0,0}, \Sigma_{1}^{0}-I A, \Delta_{1}^{0}-C A$.

2) $E \widehat{-H} A^{\omega} \uparrow+A C-\mathrm{qf} \vdash b-A C-\mathrm{qf}$ (Analogous for $E-H A^{\omega}$ ).

\section{Proof:}

1) is standard.

2) follows from $E \widehat{-H} A^{\omega} \wedge \vdash \exists y \leq_{\rho} x A_{0}(x, y) \leftrightarrow \exists y A_{0}\left(x, \min _{\rho}(x, y)\right)$.

\footnotetext{
${ }^{2} x^{\prime}$ stands for $S x$ where $S$ is the successor function.
} 


\section{Extraction of uniform bounds in higher types}

\subsection{Notational conventions}

1) In the following $r, s, t$ and $\Phi, \Psi$ always denote closed terms of Gdel's calculus $T$ or Kleene/Feferman's calculus $\widehat{P R}$ of primitive recursive functionals of finite type as defined in 1.

2) ¿From now on (up to the end of this paper) all free variables of formulas are indicated, i.e. expressions of the form " $\forall u ; v \leq t u\left(\forall x \exists y \leq s u v x \forall z A_{0}(u, v, x, y, z) \rightarrow \exists w^{2} B_{0}(u, v, w)\right)$ always stay for closed formulas (which are called sentences as usual). Sometimes we abbreviate e.g. $\forall x \exists y \forall z A(x, y, z)$ by $\forall x \exists y \forall z A$.

¿From Kohlenbach (1992) we recall the following definition of pointwise strong majorization, which is a variant of notions due to W.A. Howard (1973) and M. Bezem (1985).

\subsection{Definition}

For $x^{*}, x$ of type $\rho, x^{*} \operatorname{maj}_{\rho} x$ is given by

$$
\left\{\begin{array}{l}
x^{*} \operatorname{maj}_{0} x: \equiv x^{*} \geq x \\
x^{*} \operatorname{maj}_{\rho 0} x: \equiv \forall n^{0}\left(x^{*} n \operatorname{maj}_{\rho} x n\right), \\
x^{*} \operatorname{maj}_{\rho \tau} x: \equiv \forall y^{*}, y\left(y^{*} \operatorname{maj}_{\tau} y \rightarrow x^{*} y^{*} \operatorname{maj}_{\rho} x^{*} y, x y\right) \\
(\tau \neq 0)
\end{array}\right.
$$

Here " $\geq$ " denotes the usual primitive recursively defined inequality relation for objects of type 0 . A discussion of the basic properties of $\operatorname{maj}_{\rho}$ can be found in Kohlenbach (1992).

The following theorem shows how one can extract bounds for existence quantifiers of type $\leq$ 2(not depending on bounded parameters), which are proved from premises of the form $\forall x^{\delta} \exists y \leq_{\rho}$ $s x \forall z^{\tau} A_{0}(x, y, z)$ :

\subsection{Theorem}

1) $W E-P A^{\omega}+A C-\mathrm{qf} \vdash \forall u^{1} ; v \leq_{\gamma} t u\left(\forall x^{\delta} \exists y \leq_{\rho} \operatorname{suv} x \forall z^{\tau} A_{0}(u, v, x, y, z) \rightarrow \exists w^{2} B_{0}(u, v, w)\right)$

$\Rightarrow \exists \Phi^{21} \in T$ such that

$$
\begin{array}{r}
W E-H A^{\omega}+b-A C^{\delta, \rho}-\forall \vdash \forall u ; v \leq_{\gamma} t u\left(\forall x^{\delta} \exists y \leq_{\rho} \operatorname{suvx} \forall z^{\tau} A_{0}(u, v, x, y, z)\right. \\
\left.\rightarrow \exists w \leq_{2} \Phi u B_{0}(u, v, w)\right) .
\end{array}
$$

$\Phi$ can be extracted from any given proof of the assumption by functional interpretation combined with majorization.

2) The systems $W E-P A^{\omega}, T$ and $W E-H A^{\omega}$ in 1) can be replaced by $W \widehat{E-P A^{\omega}}, \widehat{P R}$ and $W \widehat{E-H} H A^{\omega} \uparrow$. 


\section{Proof:}

1) $W E-P A^{\omega}+A C-\mathrm{qf} \vdash \forall u^{1} ; v \leq_{\gamma} t u\left(\forall x^{\delta} \exists y \leq_{\rho}\right.$ suv $\left.\forall \forall z^{\tau} A_{0} \rightarrow \exists w^{2} B_{0}\right) \Rightarrow$ $W E-P A^{\omega}+A C-\mathrm{qf} \vdash \forall u^{1} ; v \leq_{\gamma} t u\left(\exists Y \leq_{\rho \delta} \operatorname{suv} \forall x^{\delta}, z^{\tau} A_{0}(u, v, x, Y x, z) \rightarrow \exists w B_{0}\right) \Rightarrow$ $W E-P A^{\omega}+A C-\mathrm{qf} \vdash \forall u^{1} ; v \leq_{\gamma} t u ; Y \leq_{\rho \delta} \operatorname{suv} \exists x, z, w\left(A_{0} \rightarrow B_{0}\right)$.

By functional interpretation (see Kohlenbach $(1992), 3.3$ ) one extracts a closed term $\Phi_{0} \in T$ such

that

$$
(+) W E-H A^{\omega} \vdash \forall u^{1} ; v \leq_{\gamma} t u ; Y \leq \operatorname{suv}\left(\forall x, z A_{0} \rightarrow B_{0}\left(u, v, \Phi_{0} u v Y\right)\right) \text {. }
$$

By Kohlenbach (1992) 2.15, one can construct closed terms $\Phi_{0}^{*}, s^{*}, t^{*} \in T$ with

$$
W E-H A^{\omega} \vdash \Phi_{0}^{*} \operatorname{maj} \Phi_{0} \wedge s^{*} \operatorname{maj} s \wedge t^{*} \operatorname{maj} t .
$$

Define $\Phi:=\lambda u^{1} \cdot \Phi_{0}^{*} u\left(t^{*} u\right)\left(s^{*} u\left(t^{*} u\right)\right) \in T$ if $\gamma>0$ and $\Phi:=\lambda u^{1} \cdot\left(\Phi_{0}^{*} u\right)^{M}\left(t^{*} u\right)\left(\left(s^{*} u\right)^{M}\left(t^{*} u\right)\right)$ if $\gamma=0$, where $\left(x^{\rho 0}\right)^{M}=\lambda n . \max _{\rho}(x 0, \ldots, x n)$ (see Kohlenbach (1992) 2.11). As in the proof of 3.1.1 in Kohlenbach (1992) one shows that

$$
W E-H A^{\omega} \vdash \forall u ; v \leq t u ; Y \leq \operatorname{suv}\left(\Phi u m a j_{2} \Phi_{0} u v Y\right)
$$

which implies

$$
(++) W E-H A^{\omega} \vdash \forall u ; v \leq t u ; Y \leq \operatorname{suv}\left(\Phi u \geq_{2} \Phi_{0} u v Y\right)(\text { Kohlenbach (1992),2.5.2) }
$$

$(+)$ and $(++)$ imply that

$$
W E-H A^{\omega} \vdash \forall u ; v \leq t u ; Y \leq \operatorname{suv}\left(\forall x, z A_{0} \rightarrow \exists w \leq_{2} \Phi u B_{0}(u, v, w)\right)
$$

Hence

$$
W E-H A^{\omega} \vdash \forall u ; v \leq t u\left(\exists Y \leq s u v \forall x, z A_{0}(u, v, x, Y x, z) \rightarrow \exists w \leq_{2} \Phi u B_{0}\right) .
$$

Using $b-A C^{\delta, \rho}-\forall$ one concludes

$$
W E-H A^{\omega}+b-A C^{\delta, \rho}-\forall \vdash \forall u ; v \leq t u\left(\forall x \exists y \leq \operatorname{suv} \forall \forall z A_{0} \rightarrow \exists w \leq_{2} \Phi u B_{0}\right) .
$$

2) is proved similar using the analogous result for $\widehat{P R}$ proved in Kohlenbach (1992). 


\subsection{Corollary to the proof of 2.3}

1) If the quantifier " $\forall x$ " does not occur then the conclusion can be proved without $b-A C$ :

$$
\left\{\begin{array}{l}
W E-P A^{\omega}+A C-\mathrm{qf} \vdash \forall u^{1} ; v \leq_{\gamma} \operatorname{tu}\left(\exists y \leq_{\rho} \operatorname{suv} \forall z^{\tau} A_{0} \rightarrow \exists w^{2} B_{0}\right) \\
\Rightarrow \exists \Phi^{21} \in T \text { such that } \\
W E-H A^{\omega} \vdash \forall u^{1} ; v \leq_{\gamma} t u\left(\exists y \leq_{\rho} \operatorname{suv} \forall z A_{0} \rightarrow \exists w \leq_{2} \Phi u B_{0}\right) .
\end{array}\right.
$$

2) For variables $w^{\lambda}$ of an arbitrary type $\lambda, 2.3$ holds with " $\exists w\left(\Phi u \operatorname{maj}_{\lambda} w \wedge B_{0}(u, v, w)\right)$ " instead of " $\exists w \leq_{\lambda} \Phi u B_{0}(u, v, w)$ ". If one has " $\exists w^{2}, \tilde{w}^{\lambda} B_{0}(u, v, w, \tilde{w})$ " instead of " $\exists w^{2} B_{0}$ " then it is still possible to bound " $\exists w^{2}$ " by " $\exists w \leq_{2} \Phi u \exists \tilde{w} B_{0}$ ".

3) 2.3 holds also if $\operatorname{grad}(\operatorname{type} / w) \leq 2$, where $\operatorname{grad}(\rho)$ is defined by $\operatorname{grad}(0):=0, \operatorname{grad}(\rho \tau):=\max (\operatorname{grad}(\rho), \operatorname{grad}(\tau)+1)$.

Furthermore, 2.3. generalizes to tuples $\underline{u}, \underline{v}, \underline{z}, \underline{w}$ of variables $\left(\operatorname{grad}\left(u_{i}\right) \leq 1, \operatorname{grad}\left(w_{i}\right) \leq 2\right)$ and (with a corresponding modification of $b-A C-\forall$ ) also for tuples $\underline{x}, \underline{y}$ instead of the single variables $u, v, z, w, x, y$.

4) The theorem generalizes immediately to the situation where one has a finite conjunction of premises having the form $\forall x^{\delta} \exists y \leq_{\rho} \operatorname{suv} \forall \forall z^{\tau} A_{0}$.

\subsection{Corollary to 2.3}

1) $W E-P A^{\omega}+A C-\mathrm{qf} \vdash \forall u^{1} ; v \leq_{\gamma} t u\left(\forall x^{\delta} \exists y \leq_{\rho} \operatorname{suv} \forall \forall z^{\tau} A_{0}(u, v, x, y, z)\right.$

$\Longrightarrow \exists \tilde{\Phi}^{0 \gamma 1} \in T$ such that

$$
\left.\rightarrow \exists w^{0} B_{0}(u, v, w)\right)
$$

$W E-H A^{\omega}+b-A C^{\delta, \rho}-\forall \vdash \forall u ; v \leq_{\gamma} t u\left(\forall x^{\delta} \exists y \leq_{\rho} \operatorname{suv} x \forall z^{\tau} A_{0} \rightarrow B_{0}(u, v, \tilde{\Phi} u v)\right)$.

In particular, if $\Gamma$ is a set of sentences having the form

$\forall x^{\delta} \exists y \leq_{\rho} s x \forall z^{\tau} A_{0}(x, y, z)$ then the following rule holds

$$
\left\{\begin{array}{l}
W E-P A^{\omega} \oplus A C-\mathrm{qf} \oplus \Gamma \vdash \forall u^{1} \exists w^{0} B_{0}(u, w) \Rightarrow \exists \Phi^{01} \in T: \\
W E-H A^{\omega} \oplus \Gamma \oplus b-A C-\forall \vdash \forall u B_{0}(u, \Phi u) .
\end{array}\right.
$$

2) Analogous for $W \widehat{E-P} A^{\omega} \wedge, \widehat{P R}$ and $W \widehat{E-} H A^{\omega} \wedge$ instead of $W E-P A^{\omega}, T$ and $W E-$ $H A^{\omega}$.

\section{Proof:}

1) Using 2.3 one gets a bound $\Phi \in T$ such that $\exists w \leq \Phi u B_{0}(u, v, w)$. Since $B_{0}$ is quantifier-free, there exists a closed term $\chi_{B_{0}} \in T$ with

$W E-H A^{\omega} \vdash \forall u, v, w\left(\chi_{B_{0}} u v w=_{0} 0 \leftrightarrow B_{0}(u, v, w)\right)$ 
(see e.g. Luckhardt (1973) or Troelstra (1973). Define $\tilde{\Phi} \in T$ such that

$$
\tilde{\Phi} u v=\left\{\begin{array}{l}
\min w \leq_{0} \Phi u\left[\chi_{B_{0}} u v w={ }_{0} 0\right] \text { if such a } w \text { exists } \\
0^{0} \text { otherwise. }
\end{array}\right.
$$

Since $\Phi, \chi_{B_{0}} \in T$ it follows that such a $\tilde{\Phi} \in T$ exists. $\tilde{\Phi}$ fulfils the claim.

Now assume $W E-P A^{\omega} \oplus A C-\mathrm{qf} \oplus \Gamma \vdash \forall u^{1} \exists w^{0} B_{0}(u, v)$. There exist finitely many sentences $A_{1}, \ldots, A_{n} \in \Gamma$ such that

$$
W E-P A^{\omega} \oplus A C-\mathrm{qf} \oplus A_{1} \oplus \ldots \oplus A_{n} \vdash \forall u^{1} \exists w^{0} B_{0} .
$$

Hence

$$
W E-P A^{\omega} \oplus A C-\mathrm{qf} \vdash \bigwedge_{i=1}^{n} A_{i} \rightarrow \forall u \exists w B_{0} .
$$

The corollary now follows by the reasoning above together with 2.4.4.

2) follows analogously since $\widehat{P R}$ is also closed under bounded search.

\subsection{Remarks}

1) The bound $\Phi$ in 2.3 is extracted by functional interpretation of the proof of a sentence having the form $\forall a^{1} ; b \leq_{\delta} s a \forall c \leq_{\rho} r a b \exists d^{\tau} F_{0}(a, b, c, d)$ and majorizing the resulting primitive recursive term $\Phi_{0} \in T$. As the proof of 2.15 in Kohlenbach (1992) shows, such a majorizing functional can be constructed in a quite simple manner and uses only the operation $x^{\rho 0} \mapsto x^{M}$ where $x^{M}:=\lambda n^{0} \cdot \max _{\rho}(x 0, \ldots, x n)$ besides $\Phi_{0}$. In applications to concrete mathematical examples, this construction will be done in the mathematically most natural way and not follow in detail the general procedure from the proof in Kohlenbach (1992). Also in mathematical applications the terms s,t are usually majorizable in a straightforeward way.

2) The proof of theorem 2.3 uses essentially the majorizability of primitive recursive functionals of higher types (the raising of types reduces the logical complexity of the original formula!): Even for the special case

$$
\text { (*) } \forall x^{1} \exists y \leq_{1} s x \forall z^{0} A_{0} \rightarrow \forall u^{0} \exists w^{0} B_{0} .
$$

one has to majorize a functional $\Phi_{0}$ of type 3 in order to obtain a bound for $\Phi_{0}$ on arguments $Y \leq_{1(1)} s$. While majorizability for type-2-objects follows also from the uniform continuity of primitive recursive functionals of type 2 (on bounded domains), this hereditary boundedness for types $\geq 3$ is an important property of the mathematical structure of the $T$-definable functionals which no longer holds for (proof-theoretic inessential enlargements as) $T+\mu_{1}$ (where

$\mu_{1} x^{0(00)} y^{00}:=\min n\left[x(\overline{y, n})={ }_{0} x y\right]$, see Kohlenbach (1992), or type structures as HEO or ECF (see Troelstra (1973),Kohlenbach (1990)). 
3) The use of bounded search in the definition of the algorithm $\tilde{\Phi}$ in 2.5 may be replaced by more simple operations using additional information in concrete applications, e.g. if $B_{0}$ is monotonic w.r.t $w$,i.e

$$
\forall u ; v \leq t u ; w_{1}, w_{2}\left(B_{0}\left(u, v, w_{1}\right) \wedge w_{2} \geq w_{1} \rightarrow B_{0}\left(u, v, w_{2}\right)\right)
$$

then $\tilde{\Phi}$ can be identified with $\Phi$. This is the case for an important class of examples (namely uniqueness sentences in classical analysis), where $\Phi$ is of mathematical interest since it does not depend on $v$ (this will be discussed in detail in a subsequent paper).

\subsection{Proposition}

If in $2.3 \operatorname{grad}(\rho), \operatorname{grad}(\gamma) \leq 1$ then $W E-P A^{\omega}+A C$-qf can be replaced by $E-P A^{\omega}+A C^{\alpha, \beta}-\mathrm{qf}$ where $\left(\alpha=0 \wedge \beta\right.$ arbitrary) or $(\alpha=1 \wedge \beta=0)$. In $2.5 W E-P A^{\omega} \oplus A C-\mathrm{qf} \oplus \Gamma$ can be replaced by $E-P A^{\omega}+A C^{\alpha, \beta}-\mathrm{qf}+\Gamma$ if $\Gamma$ consists of sentences of the form $\forall x^{\delta} \exists y \leq \rho s x \forall z^{\tau} A_{0}$ where $\rho, \gamma$ fulfil the above restriction. This also holds for the corresponding restricted systems.

\section{Proof:}

Assume for simplicity $\rho=\gamma=1$ and

$$
E-P A^{\omega}+A C^{\alpha, \beta}-\mathrm{qf} \vdash \forall u^{1} ; v \leq_{1} t u\left(\forall x^{\delta} \exists y \leq_{1} \operatorname{suv} x \forall z^{\tau} A_{0} \rightarrow \exists w^{2} B_{0}\right) .
$$

By elimination of extensionality (see Luckhardt (1973)) this sentence can be proved also without the axiom of extensionality, in particular it can be proved within $W E-P A^{\omega}+A C^{\alpha, \beta}$-qf. One easily verifies that the elimination procedure can also be applied to the restricted system $E \widehat{-P} A^{\omega} \uparrow$.

In the following we show that, using a more complicated extraction of the bound $\Phi$, one can prove the conclusion of 2.3 within $W E-H A^{\omega}+b-A C$-qf and $E-H A^{\omega}+A C-\mathrm{qf}$ which avoids the need of higher bounded choice.

Firstly, we need the following

\subsection{Lemma}

1) Let $A_{0} \in \mathcal{L}\left(W E-P A^{\omega}\right)$ be a quantifier-free formula (possible containing further variables than $x, y, z)$. Then the following holds:

$$
W E-P A^{\omega}+A C-\mathrm{qf} \vdash \forall z^{\rho}\left(\forall x \leq_{\rho} z \exists y^{\tau} A_{0}(x, y, z) \rightarrow \exists Y^{\tau} \forall_{x} \leq_{\rho} z A_{0}(x, Y x, z)\right) .
$$

2) Analogous for $W \widehat{E-P} A^{\omega} \uparrow$ instead of $W E-P A^{\omega}$.

\section{Proof:}

Assume $\rho=0 \rho_{k} \ldots \rho_{1}$. Then

$\forall x \leq_{\rho} z \exists y^{\tau} A_{0}(x, y, z) \rightarrow \forall x\left(\forall v_{1}^{\rho_{1}}, \ldots, v_{k}^{\rho_{k}}\left(x \underline{v} \leq_{0} z \underline{v}\right) \rightarrow \exists y^{\tau} A_{0}(x, y, z)\right)$

$\rightarrow \forall x \exists v_{1}, \ldots, v_{k}, y\left(x \underline{v} \leq_{0} z \underline{v} \rightarrow A_{0}(x, y, z)\right)$. 
The lemma now follows from the fact that $\widehat{W \widehat{E-P}} A^{\omega} \uparrow$ allows the coding of tuples $v_{1}, \ldots, v_{k}, y$ of functionals into a single functional (of suitable type, which depends on $\rho_{1}, \ldots, \rho_{k}, \tau$ only).

\subsection{Theorem}

1) $W E-P A^{\omega}+A C-\mathrm{qf} \vdash \forall u^{1} ; v \leq_{\gamma} t u\left(\forall x^{\delta} \exists y \leq_{\rho} \operatorname{suv} \forall \forall z^{\tau} A_{0}(u, v, x, y, z)\right.$

$$
\left.\rightarrow \exists w^{2} B_{0}(u, v, w)\right)
$$

$\Rightarrow \exists \Phi^{21} \in T$ such that

$W E-H A^{\omega}+b-A C-\mathrm{qf} \vdash \forall u ; v \leq_{\gamma} t u\left(\forall x^{\delta} \exists y \leq_{\rho} \operatorname{suv} \forall \forall z A_{0}(u, v, x, y, z)\right.$

$$
\left.\rightarrow \exists w \leq_{2} \Phi u B_{0}(u, v, w)\right) .
$$

The conclusion can also be proved within $E-H A^{\omega}+A C$-qf.

$\Phi$ can be extracted by functional interpretation and majorization.

2) Analogous for $W \widehat{E-P} A^{\omega} \uparrow, W \widehat{E-} H A^{\omega} \wedge, E \widehat{-H} A^{\omega} \uparrow, \widehat{P R}$.

\section{Proof:}

1) Let $\mathcal{T}$ denote $W E-P A^{\omega}+A C$-qf. The assumption implies

$$
\begin{aligned}
& \mathcal{T} \vdash \forall u ; v \leq t u\left(\forall x \neg \forall y \leq_{\rho} \operatorname{suv} x \exists z^{\tau} \neg A_{0} \rightarrow \exists w^{2} B_{0}\right) \Rightarrow(2.8) \\
& \mathcal{T} \vdash \forall u ; v \leq t u\left(\forall x \neg \exists Z^{\tau \rho} \forall y \leq_{\rho} \operatorname{suv} x \neg A_{0}(u, v, x, y, Z y) \rightarrow \exists w^{2} B_{0}\right) \Rightarrow \\
& \mathcal{T} \vdash \forall u ; v \leq t u\left(\forall x, Z^{\tau \rho} \exists y \leq_{\rho} \operatorname{suv} x A_{0}(u, v, x, y, Z y) \rightarrow \exists w^{2} B_{0}\right) \Rightarrow \\
& \mathcal{T} \vdash \forall u ; v \leq t u\left(\exists Y \leq \lambda \tilde{Z}^{\tau \rho} . \operatorname{suv} \forall x, Z^{\tau \rho} A_{0}(u, v, x, Y Z x, Z(Y Z x)) \rightarrow \exists w^{2} B_{0}\right) .
\end{aligned}
$$

By 2.4.1 one can extract a closed term $\Phi^{21} \in T$ such that

$$
W E-H A^{\omega} \vdash \forall u ; v \leq t u\left(\exists Y \leq \lambda \tilde{Z}^{\tau \rho} . s u v \forall x, Z A_{0}(u, v, x, Y Z x, Z(Y Z x)) \rightarrow \exists w \leq_{2} \Phi u B_{0}\right) .
$$

Since $\forall x, Z$ can be replaced by a single $\forall$-quantifier via coding, this implies

$$
\begin{aligned}
& W E-H A^{\omega}+b-A C-\mathrm{qf} \vdash \forall u ; v \leq t u\left(\forall x, Z \exists y \leq \operatorname{suvx} A_{0}(\ldots, Z y) \rightarrow \exists w \leq_{2} \Phi u B_{0}\right) \\
\Rightarrow & \\
& W E-H A^{\omega}+b-A C-\mathrm{qf} \vdash \forall u ; v \leq t u\left(\forall x \exists y \leq \operatorname{suv} x \forall z A_{0}(\ldots, z) \rightarrow \exists w \leq_{2} \Phi u B_{0}\right) \\
\Rightarrow & (1.3 .2) \\
& \quad E-H A^{\omega}+A C-\mathrm{qf} \vdash \forall u ; v \leq t u\left(\forall x \exists y \leq \operatorname{suv} x \forall z A_{0} \rightarrow \exists w \leq_{2} \Phi u B_{0}(u, v, w)\right) .
\end{aligned}
$$

2) is proved analogously. 


\subsection{Remark}

Using (a suitable) negative translation (e.g. the translation * from Luckhardt (1973)), the assumption of 2.9 implies

$$
(*) W E-H A^{\omega}+A C-\mathrm{qf}+(M P)^{\omega} \vdash \forall u ; v \leq t u\left(\forall x^{\delta} \neg \neg \exists y \leq \operatorname{suv} x \forall z A_{0} \rightarrow \exists w B_{0}\right) .
$$

If one treats the bounded quantifiers for the moment as usual quantifiers in the definition of functional interpretation, then functional interpretation applied to $(*)$ yields

$$
W E-H A^{\omega} \vdash \forall u ; v \leq t u ; Y \leq \lambda \tilde{Z} . s u v \exists x, Z, w\left(A_{0}(u, v, x, Y Z x, Z(Y Z x)) \rightarrow B_{0}\right),
$$

which corresponds to the reasoning in the proof of 2.9 .

On the other hand, if $(*)$ is weakened by deleting " $\neg \neg$ " in the premise, i.e.

$$
(* *) W E-H A^{\omega}+A C-\mathrm{qf}+(M P)^{\omega} \vdash \forall u ; v \leq t u\left(\forall x \exists y \leq \operatorname{suv} x \forall z A_{0} \rightarrow \exists w B_{0}\right),
$$

then functional interpretation applied directly to $(* *)$ gives

$$
W E-H A^{\omega} \vdash \forall u ; v \leq t u ; Y \leq s u v \exists x, z, w\left(A_{0}(u, v, x, Y x, z) \rightarrow B_{0}\right)
$$

as in the proof of 2.3. Therefore the difference in the extraction of $\Phi$ in 2.3 and 2.9 is due to a different use of $\neg \neg$-translation. Since the extraction in 2.3 is much easier (compared with 2.9), and since in the (most interesting) analytical case $b-A C$ can be eliminated altogether from the proof of the conclusion in 2.3 (see 3.8 ), this extraction seems to be more useful for applications.

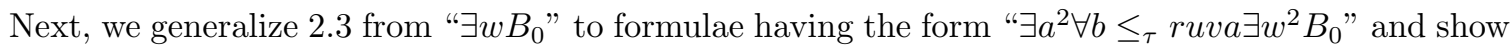
how one can extract primitive recursive bounds for $w$ and $a$. The proof uses the following

\subsection{Lemma}

1) Let $A_{0} \in \mathcal{L}\left(W E-H A^{\omega}\right)$ be a quantifier-free formula (possibly containing other free variables in addition to $\tilde{x}, \tilde{Y}, \tilde{z}, x, y, z)$. Then

$$
\begin{aligned}
E-H A^{\omega}+b-A C-\forall \vdash \forall \tilde{x}, \tilde{Y}, \tilde{z}(\forall x & \leq_{\rho} \tilde{x} \exists y \leq_{\tau} \tilde{Y} x \forall z \leq_{\delta} \tilde{z} A_{0}(\tilde{x}, \tilde{Y}, \tilde{z}, x, y, z) \\
& \left.\rightarrow \exists Y \leq_{\tau \rho} \tilde{Y} \forall x \leq_{\rho} \tilde{x} ; z \leq \tilde{z} A_{0}(\tilde{x}, \tilde{Y}, \tilde{z}, x, Y x, z)\right)
\end{aligned}
$$

2) An analogous result holds for $E \widehat{-H} A^{\omega} \uparrow$.

\section{Proof:}

1) Provable within $E-H A^{\omega}$ one has

$$
\begin{aligned}
& \forall x \leq_{\rho} \tilde{x} \exists y \leq_{\tau} \tilde{Y} x \forall z \leq_{\delta} \tilde{z} A_{0}(\tilde{x}, \tilde{Y}, \tilde{z}, x, y, z) \\
& \rightarrow \forall x \exists y \leq_{\tau} \tilde{Y}\left(\min _{\rho}(x, \tilde{x})\right) \forall z A_{0}\left(\tilde{x}, \tilde{Y}, \tilde{z}, \min _{\rho}(x, \tilde{x}), y, \min _{\delta}(z, \tilde{z})\right) .
\end{aligned}
$$


Applying $b-A C-\forall$ to the conclusion one gets

$$
\exists \widehat{Y} \leq \lambda x \cdot \tilde{Y}(\min (x, \tilde{x})) \forall x \leq \tilde{x} ; z \leq \tilde{z} A_{0}(\tilde{x}, \tilde{Y}, \tilde{z}, \min (x, \tilde{x}), Y x, \min (z, \tilde{z})) .
$$

Using extensionality the assertion in the lemma follows by putting $Y:=\min (\widehat{Y}, \tilde{Y})$.

2) The proof is analogous.

\subsection{Theorem}

1) $W E-P A^{\omega}+A C-\mathrm{qf} \vdash \forall u^{1} ; v \leq_{\gamma} t u\left(\forall x^{\delta} \exists y \leq_{\rho} \operatorname{suv} x \forall z^{\eta} A_{0} \rightarrow\right.$

$$
\left.\exists a^{2} \forall b \leq_{\tau} \operatorname{ruva} \exists w^{2} B_{0}(u, v, a, b, w)\right)
$$

$\Rightarrow \exists \Phi^{21}, \tilde{\Phi}^{21} \in T$ such that

$$
\begin{aligned}
E-P A^{\omega}+b-A C-\forall \vdash \forall u^{1} ; v & \leq_{\gamma} t u\left(\forall x \exists y \leq \operatorname{suv} x \forall z A_{0} \rightarrow\right. \\
& \left.\exists a \leq_{2} \tilde{\Phi} u \forall b \leq_{\tau} \operatorname{ruva} \exists w \leq_{2} \Phi u B_{0}(u, v, a, b, w)\right)
\end{aligned}
$$

$\Phi, \tilde{\Phi}$ can be extracted by functional interpretation and majorization.

2) 1) holds analogously for $W \widehat{E-P} A^{\omega} \uparrow, \widehat{P R}$ and $E \widehat{E-P} A^{\omega} \uparrow$.

\section{Proof:}

1) The following implications hold by logic:

$$
\begin{aligned}
& \exists a^{2} \forall b \leq_{\tau} \operatorname{ruva} \exists w^{2} B_{0}(u, v, a, b, w) \rightarrow \\
& \neg \forall a^{2} \exists b \leq_{\tau} \operatorname{ruva} \forall w^{2} \neg B_{0}(u, v, a, b, w) \rightarrow \\
& \neg \exists B \leq_{\tau 2} \operatorname{ruv} \forall a^{2}, w^{2} \neg B_{0}(u, v, a, B a, w) \rightarrow \\
& \forall B \leq_{\tau 2} \operatorname{ruv} \exists a^{2}, w^{2} B_{0}(u, v, a, B a, w) .
\end{aligned}
$$

Therefore the assumption of the theorem implies

$W E-P A^{\omega}+A C-\mathrm{qf} \vdash \forall u^{1} ; v \leq_{\gamma} t u\left(\forall x^{\delta} \exists y \leq_{\rho} s u v x \forall z^{\eta} A_{0} \rightarrow\right.$

$$
\left.\forall B \leq_{\tau 2} r u v \exists a^{2}, w^{2} B_{0}(u, v, a, B a, w)\right) .
$$


By 2.3 one can extract closed terms $\tilde{\Phi}, \Phi \in T$ such that

$W E-H A^{\omega}+b-A C-\forall \vdash \forall u^{1} ; v \leq_{\gamma} t u\left(\forall x \exists y \leq \operatorname{suv} \forall \forall z A_{0} \rightarrow\right.$

$$
\left.\forall B \leq_{\tau 2} \operatorname{ruv} \exists a \leq_{2} \tilde{\Phi} u \exists w \leq_{2} \Phi u B_{0}(u, v, a, B a, w)\right)
$$

Since

$$
\begin{aligned}
& \forall B \leq_{\tau 2} r u v \exists a \leq_{2} \tilde{\Phi} u \exists w \leq_{2} \Phi u B_{0}(u, v, a, B a, w) \rightarrow \text { (by logic) } \\
& \neg \exists B \leq_{\tau 2} r u v \forall a \leq_{2} \tilde{\Phi} u \forall w \leq_{2} \Phi u \neg B_{0}(u, v, a, B a, w) \rightarrow\left(\text { in } E-H A^{\omega}+b-A C-\forall, 2.11\right) \\
& \neg \forall a \leq_{2} \tilde{\Phi} u \exists b \leq_{\tau} r u v a \forall w \leq_{2} \Phi u \neg B_{0}(u, v, a, b, w) \rightarrow(\text { by logic }) \\
& \exists a \leq_{2} \tilde{\Phi} u \forall b \leq_{\tau} r u v a \exists w \leq_{2} \Phi u B_{0},
\end{aligned}
$$

the theorem follows.

2) is proved analogously.

In the proof of 2.3 we reduced the original situation to

$$
W E-P A^{\omega}+A C-\mathrm{qf} \vdash \forall u^{1} ; v \leq_{\gamma} t u ; Y \leq_{\rho \delta} s u v \exists x, z, w\left(A_{0} \rightarrow B_{0}\right)
$$

and constructed a bound $\Phi u$ for $w$. If the types of $x$ and $z$ are $\leq 2$ then it is also possible to bound " $\exists x$ " and " $\exists z$ ". Thus $\exists w B_{0}$ can be proved from the weakened assumption $\forall x \leq \chi u \exists y \leq \operatorname{suv} x \forall z \leq \psi u A_{0}$ for suitable $\chi, \psi \in T$. We formulate this only for " $\exists z$ " since the possibility of bounding $\mathrm{x}$ is not used in this paper:

\subsection{Theorem}

1) $W E-P A^{\omega}+A C-\mathrm{qf} \vdash \forall u^{1} ; v \leq_{\gamma} t u\left(\forall x^{\delta} \exists y \leq_{\rho} \operatorname{suv} x \forall z^{2} A_{0}(u, v, x, y, z)\right.$

$$
\left.\rightarrow \exists w^{\tau} B_{0}(u, v, w)\right)
$$

$\Rightarrow \exists \Psi^{21} \in T:$

$W E-H A^{\omega}+b-A C^{\delta, \rho}-\forall^{b} \vdash \forall u ; v \leq t u\left(\forall x \exists y \leq \operatorname{suvx} \forall z \leq_{2} \Psi u A_{0} \rightarrow \exists w B_{0}\right)$.

2) $W E-P A^{\omega}+A C-\mathrm{qf} \vdash \forall u^{1} ; v \leq_{\gamma} t u\left(\forall x^{\delta} \exists y \leq_{\rho} \operatorname{suv} \forall \forall z^{2} A_{0} \rightarrow \exists w^{2} B_{0}\right)$

$\Rightarrow \exists \Phi^{21}, \Psi^{21} \in T:$

$W E-H A^{\omega}+b-A C^{\delta, \rho}-\forall^{b} \vdash \forall u ; v \leq t u\left(\forall x \exists y \leq \operatorname{suvx} \forall z \leq_{2} \Psi u A_{0} \rightarrow \exists w \leq_{2} \Phi u B_{0}\right)$.

If type $/ w=0$ one can compute an algorithm $\tilde{\Phi}$ for $w$ which depends on $u$ and $v$ (as in 2.5). If type $/ z=0$, then $b-A C^{\delta, \rho}-\forall^{b}$ can be weakened to $b-A C^{\delta, \rho_{-} \text {qf. }}$ 
3) $W E-P A^{\omega}+A C-\mathrm{qf} \vdash \forall u^{1} ; v \leq_{\gamma} t u\left(\forall x^{\delta} \exists y \leq_{\rho} \operatorname{suv} \forall \forall z^{2} A_{0} \rightarrow \exists a^{2} \forall b \leq_{\eta} r u v a \exists w^{2} B_{0}\right)$

$\Rightarrow \exists \Phi^{21}, \tilde{\Phi}^{21}, \Psi^{21} \in T:$

$E-P A^{\omega}+b-A C-\forall^{b} \vdash \forall u ; v \leq_{\gamma} t u\left(\forall x \exists y \leq \operatorname{suv} x \forall z \leq_{2} \Psi u A_{0}\right.$

$$
\left.\rightarrow \exists a \leq_{2} \tilde{\Phi} u \forall b \leq_{\eta} r u v a \exists w \leq_{2} \Phi u B_{0}\right) .
$$

1),2) and 3) are also valid for $W \widehat{E-P} A^{\omega} \uparrow, \widehat{P R}, W \widehat{E-H} H A^{\omega} \uparrow$ and $\widehat{E-P} A^{\omega} \uparrow$.

\section{Proof:}

1) As in the proof of 2.3 it follows that

$$
W E-P A^{\omega}+A C-\mathrm{qf} \vdash \forall u ; v \leq t u ; Y \leq s u v \exists x, z, w\left(A_{0}(u, v, x, Y x, z) \rightarrow B_{0}\right) .
$$

Using functional interpretation one extracts a closed term $\Psi_{0} \in T$ such that

$$
W E-H A^{\omega} \vdash \forall u ; v \leq t u, Y \leq \operatorname{suv}\left(\forall x A_{0}\left(u, v, x, Y x, \Psi_{0} u v Y\right) \rightarrow \exists w B_{0}\right) .
$$

By a construction analogous to the one used in the proof of 2.3 one obtains a closed term $\Psi \in T$ such that

$$
W E-H A^{\omega} \vdash \forall u ; v \leq t u ; Y \leq \operatorname{suv}\left(\Psi u \geq_{2} \Psi_{0} u v Y\right) .
$$

Hence

$$
W E-H A^{\omega} \vdash \forall u ; v \leq t u\left(\exists Y \leq s u v \forall x ; z \leq_{2} \Psi u A_{0} \rightarrow \exists w B_{0}\right) .
$$

The theorem now follows by applying $b-A C^{\delta, \rho}-\forall^{b}$ to $\forall x^{\delta} \exists y \leq s u v x \forall z \leq \Psi u A_{0}$.

2) follows from the proofs of 1) and 2.3,2.5.

3) follows from the proofs of 1) and 2.12 .

\subsection{Corollary to the proof of 2.13}

1) If " $\forall z^{2} "$ and " $\forall w^{2} "$ in 2.13 are replaced by " $\forall z^{2}, \tilde{z}^{\tau}$ " and " $\forall w^{2}, \tilde{w}^{\eta}$ " where $\tau, \eta \in \tilde{T}$ are arbitrary, then it is still possible to extract primitive recursive bounds $\Psi$ and $\Phi$ for $z^{2}$ and $w^{2}$ (which depend on $u$ only).

2) A remark analogous to 2.4 holds for theorems 2.12,2.13. 


\subsection{Corollary}

$W E-P A^{\omega}+A C-\mathrm{qf} \vdash \forall u^{1} ; v \leq_{\gamma} t u\left(\forall x^{\delta} \exists y \leq_{\rho} s u v x \forall z^{2} A_{0} \rightarrow \exists w^{0} \forall f^{1} B_{0}(u, v, w, f)\right) \quad \Rightarrow$

$W E-P A^{\omega}+A C^{0,1}-\mathrm{qf}+b-A C^{\delta, \rho}-\forall^{b} \vdash \forall u ; v \leq t u\left(\forall x^{\delta}, \tilde{z}^{2} \exists y \leq_{\rho} \operatorname{suv} x \forall z \leq_{2} \tilde{z} A_{0}\right.$

$$
\left.\rightarrow \exists w^{0} \forall f^{1} B_{0}(u, v, w, f)\right) .
$$

If type $/ z=0$, then $b-A C^{\delta, \rho}-\forall^{b}$ can be

weakened to $b-A C^{\delta, \rho}-\mathrm{qf}$.

$W E-P A^{\omega}$ can be replaced by $W \widehat{E-P} A^{\omega} \wedge$ (for $A_{0}, B_{0} \in \mathcal{L}\left(W \widehat{E-P} A^{\omega} \uparrow\right)$.

\section{Proof:}

The assumption implies that

$W E-P A^{\omega}+A C-\mathrm{qf} \vdash \forall u^{1} ; v \leq_{\gamma} t u\left(\forall x^{\delta} \exists y \leq_{\rho} s u v x \forall z^{2} A_{0} \rightarrow \forall F^{1(0)} \exists w^{0} B_{0}(u, v, w, F w)\right)$.

By 2.13.1 it follows that $\exists \Psi \in T$ (since $\operatorname{grad}(1(0))=1$ ) such that 
$W E-H A^{\omega}+b-A C^{\delta, \rho}-\forall^{b} \vdash \forall u ; v \leq t u, F^{1(0)}\left(\forall x \exists y \leq \operatorname{suv} \forall \forall z \leq_{2} \Psi u F A_{0}\right.$

$$
\left.\rightarrow \exists w^{0} B_{0}(u, v, w, F w)\right)
$$

$\Rightarrow W E-H A^{\omega}+b-A C^{\delta, \rho}-\forall^{b} \vdash \forall u ; v \leq t u, F\left(\forall x, \tilde{z}^{2} \exists y \leq \operatorname{suv} x \forall z \leq_{2} \tilde{z} A_{0}\right.$

$$
\left.\rightarrow \exists w B_{0}(u, v, w, F w)\right)
$$

Since $W E-P A^{\omega}+A C^{0,1}-\mathrm{qf} \vdash \forall F^{1(0)} \exists w^{0} B_{0}(u, v, w, F w) \rightarrow \exists w^{0} \forall f^{1} B_{0}(u, v, w, f)$

the corollary follows.

\section{The analytical case}

In this paragraph we show that the conclusion of 2.13 .3 can be proved in $W E-H A^{\omega}$ (so in particular without any choice!) if all variables have types $\leq 1$.

\subsection{Primitive recursive coding and some notations}

We use the following primitive recursive coding of finite sequences of objects of type 0 :

$j(x, y):=2^{x}(2 y+1)-1, j_{1} z:=\min x \leq z\left[\exists y \leq z\left(2^{x}(2 y+1)=S z\right)\right]$,

$j_{2} z:=\min y \leq z\left[\exists x \leq z\left(2^{x}(2 y+1)=S z\right)\right]$.

$\nu_{1}(x):=x, \nu_{n+1}\left(x_{0}, x_{1}, \ldots, x_{n}\right):=j\left(x_{0}, \nu_{n}\left(x_{1}, \ldots, x_{n}\right)\right)$,

$$
j_{1}^{1}(x):=x, j_{i}^{n}(x):=\left\{\begin{array}{l}
j_{1} \circ\left(j_{2}\right)^{i-1}(x) \text { if } 1 \leq i<n \\
\left(j_{2}\right)^{n-1}(x) \text { if } 1<i=n
\end{array} \quad(\text { if } n>1) .\right.
$$

It follows that $j_{i}^{n}\left(\nu_{n}\left(x_{1}, \ldots, x_{n}\right)\right)=x_{i} \quad(1 \leq i \leq n), \nu_{n}\left(j_{1}^{n}(x), \ldots, j_{n}^{n}(x)\right)=x$.

$<>:=0,<x_{0}, \ldots, x_{n}>:=S\left(\nu_{2}\left(n, \nu_{n+1}\left(x_{0}, \ldots, x_{n}\right)\right)\right)$.

As an abbreviation we use $\widehat{x}:=<x>$. One can construct primitive recursive functions $*$, lth, $\Pi$ such that

$$
\begin{gathered}
<x_{0}, \ldots, x_{n}>*<y_{0}, \ldots, y_{m}>=<x_{0}, \ldots, x_{n}, y_{0}, \ldots, y_{m}>, \text { lth }\left(<x_{0}, \ldots, x_{n}>\right)=n+1 \\
\Pi(n, y)=\left\{\begin{array}{l}
x_{y} \text { if } y \leq m, \\
0^{0} \text { otherwise, }
\end{array} \text { for } n=<x_{0}, \ldots, x_{m}>.\right.
\end{gathered}
$$

We usually use the notation $(n)_{y}$ for $\Pi(n, y)$. For functions (i.e. functionals of type 1 ) $a^{1}$, we define $\bar{a} 0:=<>, \bar{a}(S x):=\bar{a} x *<a x>$. Thus for $x \neq 0$ one has $\bar{a} x=<a 0, \ldots, a(x-1)>$. $\bar{a} x$ is primitive recursive in $a$.

$$
\left(\bar{a} x * v^{1}\right)(y):=\left\{\begin{array}{l}
a y \text { if } y<x \\
v(y-x) \text { otherwise. }
\end{array}\right.
$$

$0^{1}:=\lambda x^{0} \cdot 0^{0}, \quad 1^{1}:=\lambda x^{0} .1^{0}$, where $1^{0}:=S 0^{0} . \overline{a, x}:=\bar{a} x * 0^{1}$. 


\subsection{Lemma}

1) Let $A_{0}\left(x^{1}, \underline{y}\right) \in \mathcal{L}\left(W E-H A^{\omega}\right)$ be a quantifier-free formula whose free variables are $x^{1}$, $\underline{y}=y_{1}, \ldots, y_{n}$ and type $/ y_{i} \leq 1(1 \leq i \leq n)$.

Then the following holds:

$$
W E-H A^{\omega} \vdash \bigwedge_{\exists} x^{1} A_{0}(x, \underline{y}) \leftrightarrow \bigwedge_{\exists} k^{0} A_{0}\left(\lambda m .(k)_{m}, \underline{y}\right) .
$$

2) An analogous result holds for $W \widehat{E-} H A^{\omega} \uparrow$ if $A_{0} \in \mathcal{L}\left(W \widehat{E-} H A^{\omega} \uparrow\right)$.

\section{Proof:}

1) There exists a closed term $t \in T$ such that

1. $W E-H A^{\omega} \vdash \forall x, \underline{y}\left(t x \underline{y}=_{0} 0 \leftrightarrow A_{0}(x, \underline{y})\right)$ (see e.g. Troelstra (1973),1.6.14).

By Troelstra (1973),2.7.8 there exists, furthermore, a primitive recursive modulus of continuity $\tilde{t} \in T$ for $t$ (w.r.t. the variable $\mathrm{x}$ ):

2. $W E-H A^{\omega} \vdash \forall x, \underline{y}, v^{1}\left(t x \underline{y}=_{0} t(\bar{x}(\tilde{t} x \underline{y}) * v) \underline{y}\right):$

1. and 2. imply

$$
\text { 3. } W E-H A^{\omega} \vdash \forall x, \underline{y}\left(A_{0}(x, \underline{y}) \leftrightarrow A_{0}\left(\bar{x}(\tilde{t} x \underline{y}) * 0^{1}, \underline{y}\right)\right) \text {. }
$$

Since $\lambda m \cdot(\bar{x}(\tilde{t} x \underline{y}))_{m}=\bar{x}(\tilde{t} x \underline{y}) * 0^{1}$, the lemma follows.

2 ) is proved analogously using the fact that each $t^{2} \in \widehat{P R}$ possesses a modulus $\tilde{t} \in \widehat{P R}$ of pointwise continuity (provable in $W \widehat{E-} H A^{\omega} \uparrow$ ), which can be shown by using an adaptation of Troelstra's proof for $T$ (see Kohlenbach (1990) for details).

\subsection{Remark}

In the proof of 3.2 we could also have used a modulus $\widehat{t} \in T(\widehat{P R})$ of uniform continuity w.r.t. $x$ for $t \in T(\widehat{P R})$, i.e.

$$
\begin{aligned}
& \text { 2. }{ }^{*} W E-H A^{\omega} \vdash \forall \tilde{x} ; x, v \leq_{1} \tilde{x} ; \underline{y}\left(\bar{v}(\widehat{t} \tilde{x} \underline{y})={ }_{0} \bar{x}(\widehat{t} \tilde{x} \underline{y}) \rightarrow t x \underline{y}={ }_{0} t v \underline{y}\right) . \\
& \left(W \widehat{E-} H A^{\omega} \uparrow\right) .
\end{aligned}
$$

Then 3. in the proof of 3.2 holds also with $\widehat{t} x \underline{y}$ instead of $\tilde{t} x \underline{y}$. Such a modulus of uniform continuity can be extracted from extensionality proofs of $t$ using functional interpretation and pointwise majorization (see Kohlenbach (1992),3.6). 


\subsection{Corollary}

1) Let $A_{0}\left(x^{0}, y^{1}\right) \in \mathcal{L}\left(W E-P A^{\omega}\right)$ be a quantifier-free formula, whose free variables are of type $\leq 1$. Then

(i) $W E-P A^{\omega}+A C^{0,0}{ }_{-\mathrm{qf}} \vdash \forall x^{0} \exists f^{1} A_{0}(x, f) \rightarrow \exists F^{1(0)} \forall x^{0} A_{0}(x, F x)$,

(ii) $W E-P A^{\omega}+A C^{0,0}-\mathrm{qf} \vdash \exists f^{1} A_{0}(0, f) \wedge \forall x^{0}\left(\exists f A_{0}(x, f) \rightarrow \exists g A_{0}\left(x^{\prime}, g\right)\right) \rightarrow \forall x \exists f A_{0}(x, f)$,

i.e. $W E-P A^{\omega}+A C^{0,0}$-qf implies $A C^{0,1}$-qf and $\exists f^{1} A_{0}$-induction for formulas $A_{0}$ having only free variables of type $\leq 1$.

2) Analogous for $W \widehat{E-P} A^{\omega} \uparrow$ instead of $W E-P A^{\omega}$.

\section{Proof:}

1)(i),2)(i) follow immediately from 3.2, and 1)(ii),2)(ii) are proved using 1.3 .1 and again 3.2.

\subsection{Lemma}

1) Let $A_{0}\left(x, \tilde{x}^{1}, y\right) \in \mathcal{L}\left(W E-H A^{\omega}\right)$ be a quantifier-free formula whose free variables are $x, \tilde{x}, \underline{y}=y_{1}, \ldots, y_{n}$ where type $/ x, \underline{y} \leq 1$. Assume that $s^{1 \delta} \in T$ is closed and

$\delta=$ type $/ x(\leq 1)$. Then there are (effectively) quantifier-free formulas $B_{0}(x, \underline{y})$ and $C_{0}(x, \underline{y})$ (containing only $x, \underline{y}$ free) such that

1. $W E-H A^{\omega} \vdash \forall \tilde{x} \leq_{1} s x A_{0}(x, \tilde{x}, \underline{y}) \leftrightarrow B_{0}(x, \underline{y})$,

2. $W E-H A^{\omega} \vdash \exists \tilde{x} \leq_{1} s x A_{0}(x, \tilde{x}, \underline{y}) \leftrightarrow C_{0}(x, \underline{y})$.

2) 1) holds also for $W \widehat{E-H} A^{\omega} \uparrow$ instead of $W E-H A^{\omega}$.

\section{Proof:}

1) As in the proof of 3.2 there exists a closed term $t \in T$ such that

$$
W E-H A^{\omega} \vdash \forall x ; \tilde{x} \leq_{1} s x ; \underline{y}\left(t x \tilde{x} \underline{y}=_{0} 0 \leftrightarrow A_{0}(x, \tilde{x}, \underline{y})\right) .
$$

By Kohlenbach (1992) $(3.5,3.6)$ one can compute a modulus $\widehat{t} \in T$ of uniform continuity for $t$ on $\left\{\tilde{x}^{1} \mid \tilde{x} \leq_{1} s x\right\}$, so in particular

$$
W E-H A^{\omega} \vdash \forall x ; \tilde{x} \leq_{1} s x ; \underline{y}\left(t x \tilde{x} \underline{y}=_{0} t x\left(\overline{\tilde{x}}(\widehat{t} x \underline{y}) * 0^{1}\right) \underline{y}\right) .
$$

It follows that

$$
W E-H A^{\omega} \vdash \bigwedge_{\exists} \tilde{x} \leq s x A_{0} \leftrightarrow \bigwedge_{\exists}^{\wedge} k^{0} \leq \Phi x \underline{y}\left\{\begin{array}{l}
\forall i<l \operatorname{thk}\left((k)_{i} \leq s x i\right) \rightarrow A_{0}\left(x, \lambda m .(k)_{m}, \underline{y}\right) \\
\forall i<l \operatorname{thk}\left((k)_{i} \leq s x i\right) \wedge A_{0}\left(x, \lambda m .(k)_{m}, \underline{y}\right),
\end{array}\right.
$$


where $\Phi \in T$ such that

$$
\Phi x \underline{y}=\max \left\{<\tilde{x}_{0}, \ldots, \tilde{x}_{\hat{t} x \underline{y}-1}>\mid \bigwedge_{i=0}^{\widehat{t} x \underline{y}-1} \tilde{x}_{i} \leq_{0} s x i\right\}
$$

There are closed terms $t_{1}, t_{2} \in T$ such that

$$
\begin{gathered}
W E-H A^{\omega} \vdash \forall x, \underline{y}\left[\left(t_{1} x \underline{y}={ }_{0} 0 \leftrightarrow \forall k \leq \Phi x \underline{y}\left(\forall i<l t h k\left((k)_{i} \leq s x i\right) \rightarrow A_{0}\left(x, \lambda m \cdot(k)_{m}, \underline{y}\right)\right)\right)\right. \\
\left.\wedge\left(t_{2} x \underline{y}={ }_{0} 0 \leftrightarrow \exists k \leq \Phi x \underline{y}\left(\forall i<l t h k\left((k)_{i} \leq s x i\right) \wedge A_{0}\left(x, \lambda m \cdot(k)_{m}, \underline{y}\right)\right)\right)\right] . \\
B_{0}(x, \underline{y}): \equiv\left(t_{1} x \underline{y}={ }_{0} 0\right) \text { and } C_{0}(x, \underline{y}): \equiv\left(t_{2} x \underline{y}={ }_{0} 0\right) \text { fulfil the lemma. }
\end{gathered}
$$

2) can be proved analogously since by Kohlenbach (1992) $(3.5,3.6)$ a modulus $\widehat{t} \in \widehat{P R}$ of uniform continuity for $t \in \widehat{P R}$ can be constructed (provable in $W \widehat{E-} H A^{\omega} \wedge$ ).

\subsection{Corollary to the proof of 3.5}

1) For each sentence of the form $\forall \underline{x}^{1} \exists y \leq_{1} s \underline{x} A_{0}(\underline{x}, y) \in \mathcal{L}\left(W E-H A^{\omega}\right)$ one can construct a closed term $\chi \in T$ such that

$$
W E-H A^{\omega} \vdash \forall \underline{x}\left(\exists y \leq s \underline{x} A_{0}(\underline{x}, y) \leftrightarrow A_{0}(\underline{x}, \chi \underline{x}) \wedge \chi \underline{x} \leq_{1} s \underline{x}\right) .
$$

2) 1. holds analogous for $W \widehat{E-} H A^{\omega} \wedge, \widehat{P R}$ instead of $W E-H A^{\omega}, T$.

\section{Proof:}

1) The proof of 3.5 yields the construction of a closed $\Phi \in T$ such that

$$
W E-H A^{\omega} \vdash \forall \underline{x}\left(\exists y \leq s x A_{0}(\underline{x}, y) \leftrightarrow \exists k \leq_{0} \Phi \underline{x}\left(A_{0}\left(\underline{x}, \lambda m .(k)_{m}\right) \wedge \forall i<l t h k\left((k)_{i} \leq s x i\right)\right)\right) .
$$

Define $\chi_{0}, \chi \in T$ such that

$$
\chi_{0} \underline{x}=\left\{\begin{array}{l}
\min k \leq \Phi \underline{x}\left[A_{0}\left(\underline{x}, \lambda m .(k)_{m}\right) \wedge \forall i<l t h k\left((k)_{i} \leq s x i\right)\right] \text { if existent } \\
0^{0} \text { otherwise, }
\end{array}\right.
$$

and $\chi \underline{x}=\lambda m \cdot\left(\chi_{0} \underline{x}\right)_{m} \cdot \quad \chi$ fulfils the claim.

2) An analogous assertion holds for $W \widehat{E-} H A^{\omega} \wedge, \widehat{P R}$ instead of $W E-H A^{\omega}, T$.

3.2 permits the construction of an algorithm for $w$ in 2.5 even when type $/ w=1$ instead of $=0$ : 


\subsection{Proposition}

1) $\left\{\begin{array}{l}W E-P A^{\omega}+A C-\mathrm{qf} \vdash \forall u^{1} ; v \leq_{1} t u\left(\forall x^{\delta} \exists y \leq_{\rho} \operatorname{suv} \forall \forall z^{\tau} A_{0} \rightarrow \exists w^{1} B_{0}(u, v, w)\right) \\ \Rightarrow \exists \tilde{\Phi}^{011} \in T \text { such that } \\ W E-H A^{\omega}+b-A C^{\delta, \rho}-\forall \vdash \forall u^{1} ; v \leq_{1} t u\left(\forall x^{\delta} \exists y \leq_{\rho} \operatorname{suv} x \forall z^{\tau} A_{0} \rightarrow B_{0}\left(u, v, \lambda m .(\tilde{\Phi} u v)_{m}\right)\right) .\end{array}\right.$

2) 1) holds also for $W \widehat{E-P A} A^{\omega} \uparrow, \widehat{P R}$ and $W \widehat{E-H} H A^{\omega} \uparrow$.

\section{Proof:}

By 3.2 one can replace " $\exists w^{1} B_{0}(u, v, w)$ " by " $\exists k^{0} B_{0}\left(u, v, \lambda m .(k)_{m}\right)$ ". The conclusion now follows from 2.5 .

\subsection{Theorem}

Assume that $(\alpha=0 \wedge \beta$ arbitrary) or $(\alpha=1 \wedge \beta=0)$.

1) $E-P A^{\omega}+A C^{\alpha, \beta}-\mathrm{qf} \vdash \forall u^{1} ; v \leq_{1} t u\left(\forall x^{1} \exists y \leq_{1} \operatorname{suv} x \forall z^{1} A_{0} \rightarrow\right.$

$$
\left.\exists a^{1} \forall b \leq_{1} \operatorname{ruva} \exists w^{1} B_{0}(u, v, a, b, w)\right)
$$

$\Rightarrow \exists \Phi, \tilde{\Phi}, \Psi \in T$ such that

$$
\begin{aligned}
& W E-H A^{\omega} \vdash \forall u^{1} ; v \leq_{1} t u\left(\forall x^{1} \exists y \leq_{1} \operatorname{suvx} \bigwedge_{i=0}^{\Psi u} A_{0}\left(u, v, x, y, \lambda m .(i)_{m}\right) \rightarrow\right. \\
&\left.\bigvee_{j=0}^{\tilde{\Phi} u} \forall b \leq_{1} \operatorname{ruv}\left(\lambda m \cdot(j)_{m}\right) \bigvee_{k=0}^{\Phi u} B_{0}\left(u, v, \lambda m \cdot(j)_{m}, b, \lambda m .(k)_{m}\right)\right) .
\end{aligned}
$$

2) Analogous for $E \widehat{E-P} A^{\omega} \uparrow, \widehat{P R}$ and $W \widehat{E-H} H A^{\omega} \uparrow$ instead of $E-P A^{\omega}, T$ and $W E-H A^{\omega}$. $\Phi, \tilde{\Phi}, \Psi$ can be extracted by functional interpretation combined with majorization.

\section{Proof:}

1) By the elimination of extensionality (see Luckhardt (1973)) and 3.2 the assumption implies

$$
\begin{gathered}
W E-P A^{\omega}+A C^{\alpha, \beta}-\mathrm{qf} \vdash \\
\forall u^{1} ; v \leq_{1} t u\left(\forall x^{1} \exists y \leq_{1} \operatorname{suv} \forall i A_{0}\left(u, v, x, y, \lambda m .(i)_{m}\right) \rightarrow\right. \\
\left.\exists a^{1} \forall b \leq_{1} \operatorname{ruva} \exists k^{0} B_{0}\left(u, v, a, b, \lambda m .(k)_{m}\right)\right) .
\end{gathered}
$$

As in the proof of 2.12 .1 one shows

$$
\begin{aligned}
W E-P A^{\omega}+A C^{\alpha, \beta}-\mathrm{qf} & \vdash u ; v \leq t u\left(\forall x^{1} \exists y \leq_{1} \operatorname{suv} x \forall i A_{0} \rightarrow\right. \\
\forall B & \left.\leq_{11} \operatorname{ruv} \exists a^{1}, k^{0} B_{0}\left(u, v, a, B a, \lambda m .(k)_{m}\right)\right) .
\end{aligned}
$$


By the proofs of 2.3 and 2.13 one can extract closed terms $\Phi, \widehat{\Phi}, \Psi \in T$ such that

$(*) W E-H A^{\omega} \vdash \forall u ; v \leq t u\left(\exists Y \leq_{11} \operatorname{suv} \forall x^{1} ; i \leq_{0} \Psi u A_{0}\left(u, v, x, Y x, \lambda m .(i)_{m}\right) \rightarrow\right.$

$$
\left.\forall B \leq_{11} r u v \exists a \leq_{1} \widehat{\Phi} u ; k \leq_{0} \Phi u B_{0}\left(u, v, a, B a, \lambda m .(k)_{m}\right)\right) .
$$

Claim:

(i) $W E-H A^{\omega} \vdash \forall x^{1} \exists y \leq_{1} \operatorname{suv} x \forall i \leq_{0} \Psi u A_{0}\left(u, v, x, y, \lambda m .(i)_{m}\right) \rightarrow$

$$
\exists Y \leq_{11} \operatorname{suv} \forall x^{1} ; i \leq_{0} \Psi u A_{0}\left(u, v, x, Y x, \lambda m .(i)_{m}\right) .
$$

(ii) $W E-H A^{\omega} \vdash \forall u ; v \leq t u\left(\forall B \leq{ }_{11} r u v \exists a \leq_{1} \widehat{\Phi} u ; k \leq_{0} \Phi u B_{0}\left(u, v, a, B a, \lambda m .(k)_{m}\right) \rightarrow\right.$

$$
\left.\exists j \leq_{0} \tilde{\Phi} u \forall b \leq \operatorname{ruv}\left(\lambda m \cdot(j)_{m}\right) \exists k \leq_{0} \Phi u B_{0}\left(u, v, \lambda m \cdot(j)_{m}, b, \lambda m .(k)_{m}\right)\right),
$$

for some closed $\tilde{\Phi} \in T$ which can be extracted from the given data.

Proof of the claim:

(i) There exists a closed $t_{A_{0}} \in T$ such that

$$
W E-H A^{\omega} \vdash t_{A_{0}} u v x y={ }_{0} 0 \leftrightarrow \forall i \leq_{0} \Psi u A_{0}\left(u, v, x, y, \lambda m .(i)_{m}\right) .
$$

Now applying 3.6 to " $t_{A_{0}} u v x y=0$ " we find a $\chi \in T$ such that

$$
\begin{gathered}
W E-H A^{\omega} \vdash \exists y \leq_{1} \operatorname{suvx}\left(t_{A_{0}} u v x y={ }_{0} 0\right) \rightarrow t_{A_{0}} u v x(\chi u v x)==_{0} 0 \wedge \chi u v x \leq_{1} \text { suvx } \Rightarrow \\
W E-H A^{\omega} \vdash \forall x \exists y \leq_{1} \operatorname{suvx}\left(t_{A_{0}} u v x y={ }_{0} 0\right) \rightarrow \forall x\left(t_{A_{0}} u v x(\chi u v x)={ }_{0} 0 \wedge \chi u v x \leq \operatorname{suv} x\right) \Rightarrow \\
W E-H A^{\omega} \vdash \forall x \exists y \leq_{1} \operatorname{suv} x\left(t_{A_{0}} u v x y=0\right) \rightarrow \exists Y^{11}\left(\forall x\left(Y x \leq_{1} \text { suvx }\right)\right. \\
\left.\wedge \forall x\left(t_{A_{0}} u v x(Y x)=0\right)\right)
\end{gathered}
$$

$\Rightarrow \quad(\mathrm{i})$

(ii) $W E-P A^{\omega} \vdash \forall B \leq_{11} r u v \exists a \leq_{1} \widehat{\Phi} u ; k \leq_{0} \Phi u B_{0}\left(u, v, a, B a, \lambda m .(k)_{m}\right) \rightarrow$

$$
\begin{gathered}
\neg \exists B \leq_{11} \text { ruv } \forall a \leq_{1} \widehat{\Phi} u ; k \leq_{0} \Phi u \neg B_{0}\left(u, v, a, B a, \lambda m(k)_{m}\right) \stackrel{(!)}{\rightarrow} \\
\neg \forall a \leq_{1} \widehat{\Phi} u \exists b \leq_{1} \text { ruva } \forall k \leq_{0} \Phi u \neg B_{0}\left(u, v, a, b, \lambda m .(k)_{m}\right) \rightarrow \\
(* *) \exists a \leq_{1} \widehat{\Phi} u \forall b \leq_{1} \text { ruva } \exists k \leq_{0} \Phi u B_{0}\left(u, v, a, b, \lambda m .(k)_{m}\right) .
\end{gathered}
$$


Ad !: The implication follows analogously to the proof of claim (i).

Let $t_{B_{0}} \in T$ be such that

$$
W E-H A^{\omega} \vdash t_{B_{0}} u v a b={ }_{0} 0 \leftrightarrow \exists k \leq_{0} \Phi u B_{0}\left(u, v, a, b, \lambda m .(k)_{m}\right) .
$$

3.5 applied to $\forall b \leq \operatorname{ruva}\left(t_{B_{0}} u v a b={ }_{0} 0\right)$ yields a closed term $\tilde{t}_{B_{0}} \in T$ such that

$$
W E-H A^{\omega} \vdash \forall b \leq \operatorname{ruva}\left(t_{B_{0}} u v a b={ }_{0} 0\right) \leftrightarrow \tilde{t}_{B_{0}} u v a={ }_{0} 0 .
$$

Let $\widehat{\tilde{t}} u \in T$ be a modulus of uniform continuity of $\tilde{t}_{B_{0}}$ on $\left\{a \mid a \leq_{1} \widehat{\Phi} u\right\}$ and $\left\{v \mid v \leq_{1} t u\right\}$ (see Kohlenbach (1992),3.5,3.6). Then

$W E-H A^{\omega} \vdash \forall v \leq_{1} t u\left(\exists a \leq_{1} \widehat{\Phi} u\left(\tilde{t}_{B_{0}} u v a=_{0} 0\right) \rightarrow \exists j^{0}(l t h j \leq \widehat{\tilde{t}} u \wedge\right.$

$$
\left.\left.\forall m<\operatorname{lth} j\left((j)_{m} \leq \widehat{\Phi} u m\right) \wedge \tilde{t}_{B_{0}}\left(u, v, \lambda m \cdot(j)_{m}\right)={ }_{0} 0\right)\right) .
$$

Since $l t h j \leq \widehat{\tilde{t}} u \wedge \forall m<\operatorname{lth} j\left((j)_{m} \leq \widehat{\Phi} u m\right)$ implies $j \leq_{0}<\widehat{\Phi} u 0, \ldots, \widehat{\Phi} u(\widehat{\tilde{t}} u-1)>=: \tilde{\Phi} u$, it follows that

$$
W E-H A^{\omega} \vdash \forall v \leq t u\left(\exists a \leq_{1} \widehat{\Phi} u\left(\tilde{t}_{B_{0}}(u, v, a)=0\right) \rightarrow \exists j \leq_{0} \tilde{\Phi} u\left(\tilde{t}_{B_{0}}\left(u, v, \lambda m .(j)_{m}\right)=0\right)\right) .
$$

Combining this with $(* *)$, we have

$(* * *) W E-P A^{\omega} \vdash \forall u ; v \leq t u\left(\forall B \leq r u v \exists a \leq \widehat{\Phi} u ; k \leq_{0} \Phi u B_{0} \rightarrow \exists j \leq_{0} \tilde{\Phi} u\left(\tilde{t}_{B_{0}}\left(u, v, \lambda m .(j)_{m}\right)=0\right)\right)$,

where

$$
\begin{aligned}
& W E-H A^{\omega} \vdash \tilde{t}_{B_{0}}\left(u, v, \lambda m \cdot(j)_{m}\right)=0 \\
& \forall b \leq_{1} \operatorname{ruv}\left(\lambda m \cdot(j)_{m}\right) \exists k \leq_{0} \Phi u B_{0}\left(u, v, \lambda m \cdot(j)_{m}, b, \lambda m \cdot(k)_{m}\right) .
\end{aligned}
$$

Applying negative translation we conclude that $(* * *)$ is provable in $W E-H A^{\omega}$, which implies (ii).

End of the proof of the claim.

The theorem follows immediately from $(*)$ and the claim.

2 ) is proved analogously.

The proof of 3.8 easily generalizes to tuples of variables (with types $\leq 1$ ) instead of the single variables $u, v, x, y, z, a, b, w$. 


\section{Weak Knig's lemma}

\subsection{Definition (WKL, Troelstra (1974))}

The weak Knig's lemma is defined to be

$$
\begin{aligned}
& W K L: \quad \forall f^{1}\left(T f \wedge \forall x^{0} \exists n^{0}(\text { lth } n=x \wedge f n=0) \rightarrow \exists b \leq_{1} \lambda k .1 \forall x^{0}(f(\bar{b} x)=0)\right), \\
& \text { where } T f: \equiv \forall n, m(f(n * m)=0 \rightarrow f n=0) \wedge \forall n, x(f(n *<x>)=0 \rightarrow x \leq 1) .
\end{aligned}
$$

Tf asserts that $f$ represents a 0,1-tree.

(The designation "weak Knig's lemma $(W K L)$ " is due to H. Friedman).

$W K L$ is equivalent (relative to $W \widehat{E-P} A^{\omega} \uparrow$ ) to the variant $W K L^{*}$, where the knots of the tree are bounded by an arbitrary function $h$ instead of $\lambda k .1$ (see 4.12 and 4.13 below).

On the other hand, the "full" Knig's lemma $K L$ is the strengthening of $W K L$, obtained when only the number of branchings (i.e. the number of successor knots to each knot) in the tree is bounded (The knots themselves, which are represented by natural numbers, are not bounded). It is known that $K L$ is equivalent to the schema of arithmetical comprehension $A C A$ (relative to $W \widehat{E-P} A^{\omega} \wedge+A C$-qf; see Friedman (1975)). This equivalence also holds if $K L$ is restricted to trees with at most two branchings). It follows that $W E-P A^{\omega}+A C$-qf $+K L$ is proof-theoretically stronger then $P A$ (see Feferman (1977),5.5.2), but $W \widehat{E-P} A^{\omega} \uparrow+A C-\mathrm{qf}+K L$ has the same strenght as PA (Shoenfield (1954), Feferman (1977),5.5.1).

If arbitrary (logically complex) formulas for the definition of 0,1 -trees are allowed in $W K L$, then the resulting strengthening of $W K L$ also implies $A C A$ (see Troelstra (1974)).

These results contrast with the conservation results for $W K L$ given below.

A detailed discussion of $W K L$ and $K L$ can be found in Kreisel/Mints/Simpson (1975).

$W K L$ is equivalent to

$$
(+) \forall f, g\left(T f \wedge \forall x(\operatorname{lth}(g x)=x \wedge f(g x)=0) \rightarrow \exists b \leq_{1} \lambda k \cdot 1 \forall x^{0}(f(\bar{b} x)=0)\right) .
$$

Since $\forall x^{0} \exists n^{0}(l t h n=x \wedge f n=0) \stackrel{\mathrm{T}(\mathrm{f})}{\rightarrow} \forall x \exists n \leq \overline{1^{00}} x(l t h n=x \wedge f n=0)$ the proof of this equivalence needs no $A C-\mathrm{qf}$.

$(+)$ is a sentence having the logical form

$$
\text { (*) } \forall x^{1}\left(\forall n^{0} A_{0}(n, x) \rightarrow \exists y \leq_{1} s x \forall z^{0} B_{0}(x, y, z)\right),
$$

where $A_{0}$ and $B_{0}$ are quantifier-free formulas. 
One could try now to generalize the results above for premises of the form $\forall x \exists y \leq s x \forall z A_{0}(x, y, z)$ directly to assumptions having the form $(*)$, in particular one can ask:

$$
(* *)\left\{\begin{array}{l}
W E-P A^{\omega}+A C-\mathrm{qf} \vdash \forall x\left(\forall n A_{0}(n, x) \rightarrow \exists y \leq_{1} s x \forall z^{0} B_{0}\right) \rightarrow \forall k^{0} \exists l^{0} C_{0} \stackrel{?}{\Rightarrow} \exists \Phi \in T: \\
W E-H A^{\omega} \vdash \forall x\left(\forall n A_{0}(n, x) \rightarrow \exists y \leq_{1} s x \forall z^{0} B_{0}\right) \rightarrow \forall k \exists l \leq \Phi k C_{0} .
\end{array}\right.
$$

This is false, however, as the following example shows (but see theorem 4.17):

Let $\forall m \exists n T_{K}(e, m, n)$ define a total recursive function, which is not definable in $T$ (here $T_{K}$ denotes Kleene's T-predicate). Classical logic yields

$$
W E-P A^{\omega} \vdash \forall m\left(\forall n \neg T_{K}(\bar{e}, m, n) \rightarrow 0=1\right) \longrightarrow \forall k \exists l T_{K}(\bar{e}, k, l) .
$$

Modulo "dummy" quantifiers, $\forall m\left(\forall n \neg T_{K}(\bar{e}, m, n) \rightarrow 0=1\right)$ has the logical form $\forall m\left(\forall n \neg T_{K}(\bar{e}, m, n) \rightarrow\right.$ $\left.\exists y \leq_{1} \lambda k \cdot 1 \forall z B_{0}(y, z)\right)$. By $(* *)$ we could extract a term $\Phi \in T$ such that $\forall k \exists l \leq \Phi k T_{K}(\bar{e}, k, l)$. But this implies that the recursive function defined by $\forall k \exists l T_{K}(\bar{e}, k, l)$ is definable in $T$, which is a contradiction.

Nevertheless the results proved so far can be applied to proofs which use $W K L$, since $W K L$ is equivalent (provable within $\left.W \widehat{E-} H A^{\omega} \uparrow\right)$ to a sentence of the form $\forall \underline{x}^{1} \exists y \leq_{1} \lambda k .1 \forall z^{0} A_{0}^{K}(\underline{x}, y, z)$ and $W \widehat{E-} H A^{\omega} \wedge \vdash \forall \underline{x}, z \exists y \leq_{1} \lambda k .1 \bigwedge_{k=0}^{z} A_{0}^{K}(\underline{x}, y, k)$ :

\subsection{Construction}

1) $\widehat{f} n:=\left\{\begin{array}{l}f n \text { if } f n \neq 0 \vee\left(\forall k, l(k * l=n \rightarrow f k=0) \wedge \forall i<l \operatorname{th} n\left((n)_{i} \leq 1\right)\right), \\ 1^{0} \text { otherwise. }\end{array}\right.$

2) $f_{g} n:=\left\{\begin{array}{l}f n \text { if } f(g(\text { lth } n))=0 \wedge l t h(g(\text { th } n))=l \text { th } n, \\ 0^{0} \text { otherwise. }\end{array}\right.$

\subsection{Remark}

$\widehat{f}\left(f_{g}\right)$ is primitive recursive in $f(f$ and $g)$ in the sense of Kleene (1952) and therefore also in the sense of $\widehat{P R}$ and $T$ ).

The operation modifies $f$ in such a way that the resulting function represents a $0,1-$ tree, i.e. $T(\widehat{f})$. If $f$ satisfies already $T f$, then doesn't change $f$ :

\subsection{Lemma}

1) $W \widehat{E-} H A^{\omega} \wedge \vdash \forall f(T(\widehat{f}))$,

2) $W \widehat{E-} H A^{\omega} \wedge \vdash \forall f\left(T(f) \rightarrow f={ }_{1} \widehat{f}\right)$.

\section{Proof:}


1) $\widehat{f}(n * m)=0 \rightarrow \widehat{f}(n * m)=f(n * m)=0 \rightarrow$

$$
\begin{aligned}
& \forall k, l(k * l=n * m \rightarrow f k=0) \wedge \forall i<l \operatorname{th}(n * m)\left((n * m)_{i} \leq 1\right) \rightarrow \\
& \forall k, l(k * l=n \rightarrow f k=0) \wedge \forall i<l \operatorname{th} n\left((n)_{i} \leq 1\right) \rightarrow \widehat{f} n=f n=0 . \\
& \widehat{f}(n *<x>)=0 \rightarrow \widehat{f}(n *<x>)=f(n *<x>)=0 \rightarrow \\
& \forall i<l \operatorname{th}(n *<x>)\left((n *<x>)_{i} \leq 1\right) \rightarrow x \leq 1 .
\end{aligned}
$$

2) Assume $T f$. Then

$$
f n=0 \rightarrow \forall k, l(k * l=n \rightarrow f k=0) \wedge \forall i<l \operatorname{th} n\left((n)_{i} \leq 1\right) .
$$

Therefore $\widehat{f} n=f n$ for all $n \in \omega$.

$f_{g}$ always satisfies the foundation condition $\forall x \exists n\left(l t h n=x \wedge f_{g} n=0\right)$. If already $\forall x(\operatorname{lth}(g x)=x \wedge f(g x)=0)$, then $f_{g}={ }_{1} f:$

\subsection{Lemma}

1) $W \widehat{E-} H A^{\omega} \wedge \vdash \forall f, g \forall x \exists n\left(l t h n=x \wedge f_{g} n=0\right)$,

2) $W \widehat{E-} H A^{\omega} \wedge \vdash \forall f, g\left(\forall x(\operatorname{lth}(g x)=x \wedge f(g x)=0) \rightarrow f_{g}={ }_{1} f\right)$.

\section{Proof:}

1) Define $n_{1}:=<\underbrace{0^{0}, \ldots, 0^{0}}_{x \text {-times }}>$. Then lth $n_{1}=x$.

Case (i): $f\left(g\left(l\right.\right.$ th $\left.\left.n_{1}\right)\right)=0 \wedge l \operatorname{th}\left(g\left(\right.\right.$ lth $\left.\left.n_{1}\right)\right)=$ th $n_{1}$ : Put $n:=g\left(\right.$ th $\left.n_{1}\right)$. Then $f(n)=0$ and therefore also $f_{g}(n)=0$.

Case (ii): $f\left(g\left(l\right.\right.$ th $\left.\left.n_{1}\right)\right) \neq 0 \vee \operatorname{lth}\left(g\left(\right.\right.$ lth $\left.\left.n_{1}\right)\right) \neq l$ th $n_{1}$ : By $f_{g}$-definition $f_{g}\left(n_{1}\right)=0$. Therefore $n:=n_{1}$ fulfils the lemma in this case.

2) $\forall x(\operatorname{lth}(g x)=x \wedge f(g x)=0) \rightarrow \forall n(f(g(\operatorname{lth} n))=0 \wedge \operatorname{lth}(g(\operatorname{lth} n))=l \operatorname{th} n)$ $\rightarrow \forall n\left(f_{g} n=f n\right)$.

\subsection{Definition}

$W K L^{\prime}: \forall f^{1}, g^{1} \exists b \leq_{1} \lambda k \cdot 1 \forall x^{0}\left(\widehat{(\widehat{f})_{g}}(\bar{b} x)={ }_{0} 0\right)$. 


\subsection{Proposition}

1) $\quad W \widehat{E-H} H A^{\omega} \wedge \vdash \forall f, g, x \exists b \leq_{1} \lambda k \cdot 1 \bigwedge_{y=0}^{x}\left(\widehat{(\widehat{f})_{g}}(\bar{b} y)={ }_{0} 0\right)$.

2) $\quad \widehat{W-} H A^{\omega} \wedge \vdash W K L \leftrightarrow W K L^{\prime}$.

\section{Proof:}

1) We show by induction on $x$

$$
(*) \forall x \exists n\left(l \text { th } n=x \wedge \forall i<x\left((n)_{i} \leq 1\right) \wedge \widehat{(\widehat{f})_{g}}(n)=0\right)
$$

(Since the quantifier " $\exists n$ " can be bounded by $\overline{1^{1}} x$, this induction is an application of (IA)-qf only).

(*) implies 1): Define $b:=n * 0^{1}$. By 4.4.1 $\widehat{(\widehat{f})_{g}}(\bar{b} x)=0$ implies $\bigwedge_{y=0}^{x} \widehat{(\widehat{f})_{g}}(\bar{b} y)=0$.

$\underline{x=0}:$ lth $n=x \leftrightarrow n=<>=0$.

Case (i): $\widehat{f}(g 0)=0 \wedge l \operatorname{th}(g 0)=0: \quad g 0=0 \wedge \widehat{f}(0)=0$.

$\widehat{f}(0)=0$ implies $(\widehat{f})_{g}(0)=0$ and furthermore $\widehat{(\widehat{f})_{g}}(0)=0$.

Case (ii): $\widehat{f}(g 0) \neq 0 \vee l t h(g 0) \neq 0: \quad(\widehat{f})_{g}(0)=0$ and therefore ${\widehat{(\widehat{f})_{g}}}_{g}(0)=0$.

$\underline{x \rightarrow x+1:}$ : By the induction hypothesis there exists a $n_{0}$ such that

lth $n_{0}=x, \widehat{(\widehat{f})_{g}}\left(n_{0}\right)=0$ and $\forall i<l$ th $n_{0}\left(\left(n_{0}\right)_{i} \leq 1\right)$.

Define $n_{1}:=n_{0} *<0>$.

Case (i): $\widehat{f}\left(g\left(l\right.\right.$ th $\left.\left.n_{1}\right)\right)=0 \wedge l \operatorname{th}\left(g\left(l\right.\right.$ th $\left.\left.n_{1}\right)\right)=l$ th $n_{1}$ :

Then $n:=g\left(l t h n_{1}\right)$ fulfils the claim:

$$
\text { lth } n=\operatorname{lth}\left(g\left(\operatorname{lth} n_{1}\right)\right)=\operatorname{lth} n_{1}=x+1 . \text { Furthermore : }
$$

$\widehat{f} n=0 \stackrel{4.4 .1}{\rightarrow} \forall k, l(n=k * l \rightarrow \widehat{f} k=0) \wedge \forall i<l \operatorname{th}\left((n)_{i} \leq 1\right)$

$$
\stackrel{4.2 .2}{\rightarrow} \forall k, l\left(n=k * l \rightarrow(\widehat{f})_{g}(k)=0\right) \wedge \forall i<l \operatorname{th}\left((n)_{i} \leq 1\right)
$$




$$
\stackrel{4.2 \cdot 1}{\rightarrow} \widehat{(\widehat{f})_{g}}(n)=0 \wedge \forall i<l \operatorname{th} n\left((n)_{i} \leq 1\right) .
$$

Case (ii): $\widehat{f}\left(g\left(l\right.\right.$ th $\left.\left.n_{1}\right)\right) \neq 0 \vee l \operatorname{th}\left(g\left(l\right.\right.$ th $\left.\left.n_{1}\right)\right) \neq l$ th $n_{1}$ : Then $n:=n_{1}$ fulfils the claim:

The case implies $(+)(\widehat{f})_{g}\left(n_{1}\right)=0$ by 4.2.2. Since $\widehat{(\widehat{f})_{g}}\left(n_{0}\right)=0$, it follows by 4.2 .1 that $\forall k, l\left(n_{0}=k * l \rightarrow(\widehat{f})_{g}(k)=0\right)$.

Together with

$(+)$ and $n_{1}=n_{0} *<0>$ this implies $\widehat{(\widehat{f})_{g}}\left(n_{1}\right)=0$.

2) “ $\rightarrow$ ": By 4.4.1, $T\left(\widehat{\left(\widehat{\hat{f})_{g}}\right)}\right.$ holds for all $f, g$.

Using 1), $W K L^{\prime}$ now follows by $W K L$.

"६": Assume $T(f)$ and $\forall x \exists n($ lth $n=x \wedge f n=0)$. Then

$$
(++) \forall x \exists n \leq \overline{1^{1}} x(\text { th } n=x \wedge f n=0) .
$$

Define

$$
g x:=\left\{\begin{array}{l}
\min n \leq \overline{1^{1}} x[l \text { th } n=x \wedge f n=0] \text { if such an } n \text { exists } \\
0^{0} \text { otherwise }
\end{array}\right.
$$

$g$ is primitive recursive in $f$ and $(++)$ implies $\forall x(l \operatorname{th}(g x)=x \wedge f(g x)=0)$. 4.5.2 yields $f_{g}={ }_{1} f$. Since $f={ }_{1} \widehat{f}(4.4 .2)$, this proves that $\widehat{(\widehat{f})_{g}}={ }_{1} f$. Using $W K L^{\prime}$ one derives $\exists b \leq_{1} \lambda k \cdot 1 \forall x^{0}(f(\bar{b} x)=0)$.

We are now able to conclude the following

\subsection{Theorem}

The results 2.3,2.4,2.5,2.7,2.9,2.12-2.15,3.7,3.8 also hold if $W E-P A^{\omega}+A C$-qf (resp. $E-P A^{\omega}+$ $A C^{\alpha, \beta_{-}}$qf) is replaced by $W E-P A^{\omega} \oplus W K L \oplus A C-\mathrm{qf}\left(\right.$ resp. $\left.E-P A^{\omega}+W K L+A C^{\alpha, \beta}-\mathrm{qf}\right)$. In particular

1) $\left\{\begin{array}{l}W E-P A^{\omega} \oplus A C-\mathrm{q} \oplus \oplus W K L \vdash \forall u^{1} ; v \leq_{\gamma} t u \exists a^{2} \forall b \leq_{\eta} r u v a \exists w^{2} A_{0}(u, v, a, b, w) \\ \Rightarrow \exists \Phi, \tilde{\Phi} \in T, \text { closed such that } \\ E-P A^{\omega}+b-A C-\forall^{b} \vdash \forall u ; v \leq_{\gamma} t u \exists a \leq_{2} \tilde{\Phi} u \forall b \leq_{\eta} r u v a \exists w \leq_{2} \Phi u A_{0} .\end{array}\right.$

2) $\left\{\begin{array}{l}W E-P A^{\omega} \oplus A C-\mathrm{qf} \oplus W K L \vdash \forall u^{1} ; v \leq_{\gamma} t u \exists w^{2} A_{0}(u, v, w) \\ \Rightarrow \exists \Phi \in T, \text { closed such that } \\ W E-H A^{\omega} \vdash \forall u ; v \leq_{\gamma} t u \exists w \leq_{2} \Phi u A_{0} .\end{array}\right.$ 


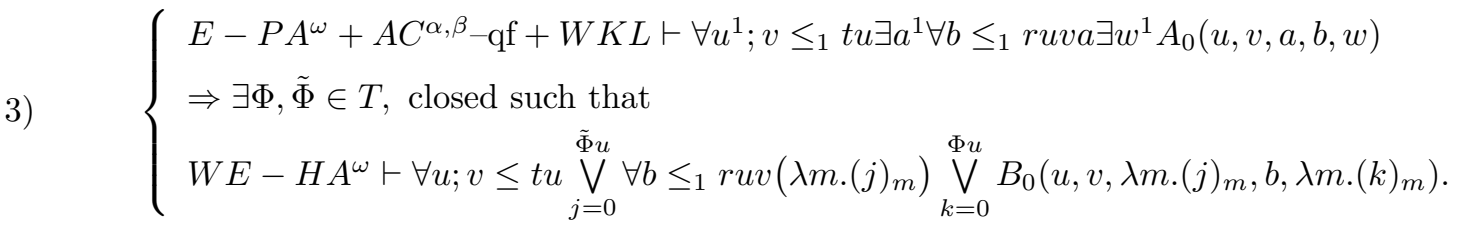

Variables of type 1 (resp. 2) can be replaced by variables of type $\delta$ with $\operatorname{grad}(\delta) \leq 1(\leq 2)$.

All the results above also hold for the corresponding restricted systems $W \widehat{E-P} A^{\omega} \uparrow, W \widehat{E-} H A^{\omega} \wedge, \widehat{E-P} A^{\omega} \wedge$ and $\widehat{P R}$.

\section{Proof:}

We prove one case of the theorem, namely that in $2.3 W E-P A^{\omega}+A C$-qf can be replaced by $W E-P A^{\omega} \oplus A C-\mathrm{qf} \oplus W K L$.

$$
\begin{aligned}
& W E-P A^{\omega} \oplus A C-\mathrm{qf} \oplus W K L \vdash \forall u^{1} ; v \leq_{\gamma} t u\left(\forall x^{\delta} \exists y \leq_{\rho} s u v x \forall z^{\tau} A_{0}(u, v, x, y, z)\right. \\
& \left.\rightarrow \exists w^{2} B_{0}(u, v, w)\right) \Longrightarrow \\
& W E-P A^{\omega} \oplus A C-\mathrm{qf} \vdash W K L \rightarrow \forall u^{1} ; v \leq_{\gamma} t u\left(\forall x^{\delta} \exists y \leq_{\rho} \operatorname{suv} x \forall z A_{0} \rightarrow \exists w^{2} B_{0}\right) \stackrel{4.7 .2}{\Longrightarrow} \\
& W E-P A^{\omega} \oplus A C-\mathrm{qf} \vdash \forall u^{1} ; v \leq_{\gamma} t u\left(\forall f, g \exists b \leq_{1} \lambda k \cdot 1 \forall n^{0}\left(\widehat{(\widehat{f})_{g}}(\bar{b} n)=_{0} 0\right)\right. \\
& \left.\wedge \forall x^{\delta} \exists y \leq_{\rho} \operatorname{suv} x \forall z A_{0} \rightarrow \exists w^{2} B_{0}\right)
\end{aligned}
$$

$\stackrel{\text { Proofof2. } 13,2.4}{\Longrightarrow} \exists \Psi, \Phi \in T:$

$$
\begin{gathered}
W E-H A^{\omega}+b-A C^{\delta, \rho}-\forall \vdash \forall u ; v \leq_{\gamma} t u\left(\exists B \leq \lambda f, g, k .1 \forall f, g \forall i \leq \Psi u\left(\widehat{(\widehat{f})_{g}}((\overline{B f g}) i)={ }_{0} 0\right)\right. \\
\left.\wedge \forall x \delta \exists y \leq_{\rho} \operatorname{suvx} \forall z A_{0} \rightarrow \exists w \leq_{2} \Phi u B_{0}\right) \stackrel{3.6}{\Longrightarrow} \\
W E-H A^{\omega}+b-A C^{\delta, \rho}-\forall \vdash \forall u ; v \leq_{\gamma} t u\left(\forall f, g \exists b \leq_{1} \lambda k \cdot 1 \forall i \leq \Psi u\left(\widehat{(\widehat{f})_{g}}(\overline{b i})=_{0} 0\right)\right. \\
\left.\wedge \forall x \exists y \leq \operatorname{suvx} \forall z A_{0} \rightarrow \exists w \leq_{2} \Phi u B_{0}\right) \Longrightarrow \\
W E-H A^{\omega}+b-A C^{\delta, \rho}-\forall \vdash \forall u ; v \leq_{\gamma} t u\left(\forall f, g, n \exists b \leq_{1} \lambda k \cdot 1 \forall i \leq n\left(\widehat{(\widehat{f})_{g}}(\overline{b i})=_{0} 0\right)\right. \\
\left.\wedge \forall x \exists y \leq \operatorname{suvx} \forall z A_{0} \rightarrow \exists w \leq_{2} \Phi u B_{0}\right) \stackrel{4.7 .1}{\Longrightarrow} \\
W E-H A^{\omega}+b-A C^{\delta, \rho}-\forall \vdash \forall u ; v \leq_{\gamma} t u\left(\forall x \exists y \leq \operatorname{suv} \forall z A_{0} \rightarrow \exists w \leq_{2} \Phi u B_{0}\right) .
\end{gathered}
$$

The other assertions in the theorem can be proved in a similar way.

Furthermore we obtain the following conservation results concerning $W K L$ : 


\subsection{Theorem}

1) $W E-P A^{\omega} \oplus A C-\mathrm{qf} \oplus W K L$ is conservative over $W E-P A^{\omega} \oplus A C^{0,1}-\mathrm{qf}\left(W E-H A^{\omega}\right)$ w.r.t. sentences of the form $\forall u^{1} ; v \leq_{\gamma} t u \exists w^{0} \forall z^{1} A_{0}\left(\forall u^{1} ; v \leq_{\gamma} t u \exists w^{\tau} A_{0}\right) \quad(\gamma, \tau \in \tilde{T}$ arbitrary).

2) $E-P A^{\omega}+A C^{\alpha, \beta}-\mathrm{qf}+W K L \quad(\alpha=0 \wedge \beta$ arbitrary $)$ or $(\alpha=1 \wedge \beta=0)$ is conservative over $W E-P A^{\omega}+A C^{0,0}{ }_{-\mathrm{qf}}\left(W E-H A^{\omega}\right)$ w.r.t. sentences of the form $\forall u^{1} ; v \leq_{1} t u \exists w^{0} \forall z^{1} A_{0} \quad\left(\forall u^{1} ; v \leq_{1} \quad t u \exists a^{1} \forall b \leq_{1} \operatorname{ruva} \exists w^{1} A_{0}\right)$

( $A_{0}$ is quantifier-free and $t, r \in T$ are closed).

Variables of type 1 can be replaced by (tuples of) variables of type $\delta$ with $\operatorname{grad}(\delta) \leq 1$.

1) and 2) also hold for the restricted systems $W \widehat{E-P} A^{\omega} \uparrow, W \widehat{E-H} H A^{\omega} \uparrow$ and $\widehat{E-P} A^{\omega} \uparrow$ instead of $W E-P A^{\omega}, W E-H A^{\omega}, E-P A^{\omega}$ (Then, of course, $t, r \in \widehat{P R}$ ).

\section{Proof:}

1) follows from 2.15 (2.13) and 4.7 (By 3.6 the proof of the conclusion does not need $b-A C-\forall^{b}$ ).

2) Assume $E-P A^{\omega}+A C^{\alpha, \beta}-\mathrm{qf}+W K L \vdash \forall u^{1} ; v \leq_{1} t u \exists w^{0} \forall z^{1} A_{0}$. Using elimination of extensionality and $3.2,4.7$ one concludes

$$
W E-P A^{\omega}+A C^{\alpha, \beta}-\mathrm{qf} \vdash W K L^{\prime} \rightarrow \forall u^{1} ; v \leq_{1} t u \exists w^{0} \forall k^{0} A_{0}\left(u, v, w, \lambda m .(k)_{m}\right) .
$$

As in 1), 2.15 and 4.7 now imply

$$
W E-P A^{\omega}+A C^{0,0}{ }_{-\mathrm{qf}} \vdash \forall u^{1} ; v \leq_{1} t u \exists w^{0} \forall k^{0} A_{0} .
$$

(Since type $/ k=0$, only $A C^{0,0}$-qf but not $A C^{0,1}$-qf is needed; the use of $b-A C-\forall^{b}$ can be avoided by 3.6).

The second assertion of 2) follows immediately from 3.8 and 4.7.

\subsection{Remark}

Theorem 4.9.1 remains valid if $W E-P A^{\omega} \oplus A C-\mathrm{qf} \oplus W K L$ and $W E-P A^{\omega} \oplus A C^{0,1}-\mathrm{qf}\left(W E-H A^{\omega}\right)$ are replaced by $W E-P A^{\omega} \oplus A C-\mathrm{qf} \oplus W K L \oplus \Gamma$ and $W E-P A^{\omega} \oplus A C^{0,1}-\mathrm{qf} \oplus \Gamma\left(W E-H A^{\omega} \oplus \Gamma\right)$, where $\Gamma$ is an arbitrary set of sentences of the form $\exists x^{1} \forall y^{\rho} F_{0}(x, y) \in \mathcal{L}\left(W E-P A^{\omega}\right)\left(F_{0}(x, y)\right.$ is quantifier-free and contains no further free variables than $x, y$ ). An analogous generalization holds for 4.9.2 (with + instead of $\oplus$ ) if $\Gamma$ is a set of sentences of the form $\exists x^{1} \forall y^{0 / 1} F_{0}(x, y)$.

This remark is also correct for the corresponding restricted systems.

\section{Proof:}

Assume

$$
W E-P A^{\omega} \oplus A C-\mathrm{qf} \oplus W K L \oplus \Gamma \vdash \forall u^{1} ; v \leq_{\gamma} t u \exists w^{0} \forall z^{1} A_{0} .
$$


Then there are finitely many sentences $F_{1}, \ldots, F_{n} \in \Gamma$ such that

$$
W E-P A^{\omega} \oplus A C-\mathrm{qf} \oplus W K L \vdash \bigwedge_{i=1}^{n} F_{i} \rightarrow \forall u^{1} ; v \leq_{\gamma} t u \exists w^{0} \forall z^{1} A_{0}
$$

For notational simplicity, we assume that $n=1$. Let $F: \equiv F_{1}: \equiv \exists x^{1} \forall y^{\rho} F_{0}(x, y)$. Then

$$
\begin{aligned}
& W E-P A^{\omega} \oplus A C-\mathrm{qf} \oplus W K L \vdash F \rightarrow \forall u^{1} ; v \leq_{\gamma} t u ; Z^{1(0)} \exists w^{0} A_{0}(u, v, w, Z w) \Rightarrow \\
& W E-P A^{\omega} \oplus A C-\mathrm{qf} \oplus W K L \vdash \forall x^{1}, Z^{1(0)} ; v \leq_{\gamma} t u \exists y^{\rho}, w\left(F_{0}(x, y) \rightarrow A_{0}(u, v, w, Z w)\right) .
\end{aligned}
$$

By 4.9.1 we conclude that

$$
\begin{aligned}
& W E-H A^{\omega} \vdash \forall x^{1}, u, Z^{1(0)} ; v \leq_{\gamma} t u \exists y, w\left(F_{0} \rightarrow A_{0}\right) \text { and therefore } \\
& W E-H A^{\omega} \vdash \exists x \forall y F_{0}(x, y) \rightarrow \forall u ; v \leq_{\gamma} t u ; Z^{1(0)} \exists w^{0} A_{0}(u, v, w, Z w), \text { which implies } \\
& W E-P A^{\omega} \oplus A C^{0,1} \text {-qf } \vdash \exists x \forall y F_{0}(x, y) \rightarrow \forall u ; v \leq_{\gamma} t u \exists w^{0} \forall z^{1} A_{0} .
\end{aligned}
$$

The other assertions of 4.10 can be proved in a similar manner.

\subsection{Remark}

$W K L$ is not conservative w.r.t. sentences of the form $(i) \exists y^{1} \forall x^{0} A_{0}(y, x)$ or $(i i) \forall x^{2} \exists y^{1} A_{0}(x, y)$ :

(i) Each instance of $W K L^{\prime}$ with $f, g \in T$ has the form $\exists y \leq_{1} \lambda k .1 \forall x^{0} A_{0}(y, x)$ and is provable in $W E-P A^{\omega} \oplus A C-\mathrm{qf} \oplus W K L$ but in general not in $W E-P A^{\omega}+A C-\mathrm{qf}$ :

Let $\exists y \leq_{1} \lambda k .1 \forall x^{0} A_{0}^{r e c}$ be the application of $W K L^{\prime}$ to the primitive recursive Kleene-tree (here $f, g \in T)$. Assume: $\mathcal{T}:=W E-P A^{\omega}+A C-\mathrm{qf}-\exists y \leq_{1} \lambda k .1 \forall x^{0} A_{0}^{r e c}$. Then

$\mathcal{T} \vdash \exists y^{1}\left(y\right.$ is not recursive) since no recursive $y$ realizes $\exists y \leq \lambda k .1 \forall x^{0} A_{0}^{\text {rec }}$ (provable within $\mathcal{T}$ ). But this contradicts the fact that $H E O \models \mathcal{T}+\forall y^{1}$ ( $y$ is recursive) (see Troelstra (1973),2.6.20,2.6.21). Analogous for $\exists y^{1} \forall x^{0} A_{0}^{\prime}$ with $A_{0}^{\prime}(y, x): \equiv A_{0}^{r e c}\left(\min _{1}(y, \lambda k .1), x\right)$.

(ii) Assume

$$
\left(W E-P A^{\omega} \oplus W K L \oplus A C-\mathrm{qf} \vdash \forall x^{2} \exists y^{1} A_{0} \Rightarrow W E-P A^{\omega}+A C-\mathrm{qf} \vdash \forall x^{2} \exists y^{1} A_{0}\right)
$$

for arbitrary quantifier-free $A_{0}$. Then

$$
\begin{aligned}
& \left(W E-P A^{\omega} \oplus W K L \oplus A C-\mathrm{qf} \vdash \exists y^{1} \forall x^{0} A_{0}(y, x) \Longrightarrow\right. \\
& W E-P A^{\omega} \oplus W K L \oplus A C-\mathrm{qf} \vdash \forall X^{2} \exists y^{1} A_{0}(y, X y) \stackrel{\text { assumption }}{\Longrightarrow} \\
& W E-P A^{\omega}+A C-\mathrm{qf} \vdash \forall X^{2} \exists y^{1} A_{0}(y, X y) \Longrightarrow \\
& \left.W E-P A^{\omega}+A C-\mathrm{qf} \vdash \exists y^{1} \forall x^{0} A_{0}(y, x)\right)
\end{aligned}
$$


but this contradicts (i).

As mentioned above, our majorant-construction allows the extraction of primitive recursive bounds (resp. algorithms) for $\forall u^{1} ; v \leq_{\gamma} t u \exists w^{0} B_{0}$-sentences from proofs, which use arbitrary assumptions of the form $\forall x^{\delta} \exists y \leq_{\rho} s x \forall z^{\tau} A_{0}$. Furthermore, weak Knig's lemma $W K L$ is equivalent to a special $\forall x^{1} \exists y \leq_{1} s x \forall z^{0} A_{0}$-sentence (namely $W K L^{\prime}$ ). Mathematical experience indicates that $\forall x^{1} \exists y \leq_{1} s x \forall z^{0} A_{0}$-sentences are quite often provable from their " $\varepsilon$-versions" using $W K L$ (e.g. the theorem that each $f \in \mathcal{C}[0,1]$ assumes its maximum on $[0,1])$. We want to prove now this universal character of $W K L$ (for the case $\delta, \rho, \tau \leq 1)$ :

Each sentence having the form $\forall x^{1} \exists y \leq_{1} s x \forall z^{1} A_{0} \in \mathcal{L}\left(W E-P A^{\omega}\right)$ follows provable in $W E-$ $P A^{\omega}+W K L$ from (*) $\forall x, k^{0} \exists y \leq s x \bigwedge_{i=0}^{k} A_{0}\left(x, y, \lambda m .(i)_{m}\right)$. By $3.5(*)$ is equivalent to a $\forall x^{1} A_{0}-$ sentence and therefore (by 3.2) to a $\forall x^{0} A_{0}$-sentence. Hence each true $\forall x^{1} \exists y \leq{ }_{1} s x \forall z^{0 / 1} A_{0}$-sentence is provable within $W E-P A^{\omega}+W K L+$ a true $\forall x^{0} A_{0}^{\prime}$-sentence.

This result is mainly of theoretical interest. For concrete extractions of bounds from given proofs it is much easier to apply our method directly to mathematical assumptions having the form $\forall x \exists y \leq s x \forall z A_{0}$ instead of first reducing them to $W K L+$ true universal sentences and then eliminating $W K L$. Furthermore, for higher types $\delta, \rho, \tau \geq 1$, it is in general not possible to reduce $\forall x^{\delta} \exists y \leq_{\rho} s x \forall z^{\tau} A_{0}$-sentences to $W K L$ plus universal sentences (see 4.16) but we still can apply our method to extract bounds from proofs which use such assumptions (as was shown in 2.3).

\subsection{Definition}

$W K L^{*}: \forall f^{1}, h^{1}\left(T(h, f) \wedge \forall x \exists n(l t h n=x \wedge f n=0) \rightarrow \exists b \leq_{1} h \forall x^{0} f(\bar{b} x)=0\right)$,

where

$$
T(f, h): \equiv \forall n, m(f(n * m)=0 \rightarrow f n=0) \wedge \forall n, x(f(n *<x>)=0 \rightarrow x \leq h(l t h(n))) .
$$

One easily proves the following lemma (using Troelstra (1973),1.9.24)

\subsection{Lemma}

$$
W \widehat{E-P} A^{\omega} \wedge \vdash W K L \leftrightarrow W K L^{*}
$$

\subsection{Proposition}

1) Let $\forall x^{1} \exists y \leq_{1} s x \forall z^{1} A_{0}(x, y, z)$ be a sentence in $\mathcal{L}\left(W E-P A^{\omega}\right)$, where $s^{11} \in T$ is a closed term. Then

$$
W E-P A^{\omega}+W K L \vdash \forall x^{1}, k^{0} \exists y \leq_{1} s x \bigwedge_{i=0}^{k} A_{0}\left(x, y, \lambda m .(i)_{m}\right) \leftrightarrow \forall x^{1} \exists y \leq_{1} s x \forall z^{1} A_{0} .
$$

2) 1) holds also for $W \widehat{E-P} A^{\omega} \wedge$ instead of $W E-P A^{\omega}$. 


\section{Proof:}

1) By 3.2 it suffices to consider $\forall x \exists y \leq s x \forall k^{0} A_{0}(x, y, k)$.

$$
\begin{aligned}
\forall x, k \exists y \leq s x \bigwedge_{i=0}^{k} A_{0}(x, y, i) \text { implies } \\
(*) \forall x, k \exists n(\underbrace{l t h n=k \wedge \forall j<k\left((n)_{j} \leq s x j\right) \wedge \exists y \leq s x \bigwedge_{i=0}^{k} A_{0}(x, n * \lambda m . y(m+k), i)}_{\overline{A_{0}}(x, k, n): \equiv}) .
\end{aligned}
$$

By 3.5 $\overline{A_{0}}$ is quantifier-free definable in $W E-P A^{\omega}$. Therefore we can define in $W E-P A^{\omega}$ a function $f_{x}$ such that

$$
f_{x} n:=\left\{\begin{array}{l}
0 \text { if } \overline{A_{0}}(x, \text { th } n, n) \\
1 \text { otherwise }
\end{array}\right.
$$

For all $x, T\left(f_{x}, s x\right)$ holds. Furthermore $(*)$ implies $\forall x, k \exists n\left(\right.$ lth $\left.n=k \wedge f_{x} n=0\right)$. Therefore $W K L^{*}$ applied to $f_{x}, s x$ yields

$$
(* *) \forall x \exists y_{0} \leq s x \forall k\left(\exists y \leq s x \bigwedge_{i=0}^{k} A_{0}\left(x, \bar{y}_{0} k * \lambda m . y(m+k), i\right)\right) .
$$

It remains to show that $\forall k A_{0}\left(x, y_{0}, k\right)$ : Assume there exists a $k \in \omega$ such that $\neg A_{0}\left(x, y_{0}, k_{0}\right)$. Since $A_{0}$ is quantifier-free, there exists a closed term $t \in T$ such that

$W E-P A^{\omega} \vdash \forall x, y, k\left(t x y k={ }_{0} 0 \leftrightarrow \neg A_{0}(x, y, k)\right)$. By Troelstra (1973),2.7.8 there exists a modulus of pointwise continuity $\tilde{t} \in T$ for $t$ w.r.t. $y$ :

$$
(* * *) W E-P A^{\omega} \vdash \forall x, y, \tilde{y}, k\left(t x(\bar{y}(\tilde{t} x y k) * \tilde{y}) k={ }_{0} t x y k\right) .
$$

Define $n_{0}:=\tilde{t} x y_{0} k_{0}$. Since $t x y_{0} k_{0}=0, \quad(* * *)$ implies $\forall m \geq n_{0}, y\left(\neg A_{0}\left(x, \bar{y}_{0} m * y, k_{0}\right)\right)$. Define $m:=\max \left(n_{0}, k_{0}\right)$, then $(* *)$ yields $\exists y \leq s x A_{0}\left(x, \bar{y}_{0} m * \lambda k \cdot y(m+k), k_{0}\right)$, which is a contradiction.

2) is proved analogously using the fact that each $t^{2} \in \widehat{P R}$ possesses a modulus of pointwise continuity $\tilde{t} \in \widehat{P R}$ provable within $W \widehat{E-P A^{\omega}} \wedge$ (see Kohlenbach (1990)).

\subsection{Corollary}

1) For each sentence of the form $\forall x^{1} \exists y \leq_{1} s x \forall z^{0 / 1} A_{0}(x, y, z) \in \mathcal{L}\left(W E-P A^{\omega}\right)$ one can construct a corresponding $\Pi_{1}^{0}$-sentence $\forall n^{0} B_{0}(n) \in \mathcal{L}\left(W E-P A^{\omega}\right)$ such that

$$
W E-P A^{\omega}+W K L \vdash \forall x^{1} \exists y \leq_{1} s x \forall z^{0 / 1} A_{0}(x, y, z) \leftrightarrow \forall n^{0} B_{0}(n) .
$$


2) An analogous result holds for $W \widehat{E-P} A^{\omega} \uparrow$ instead of $W E-P A^{\omega}$.

\section{Proof:}

The corollary follows from 4.14 together with 3.5 and 3.2 .

For types $>1$ the above corollary no longer holds:

\subsection{Proposition}

There exist true sentences $A \equiv \exists x \leq_{2} 1^{2} \forall y^{1} A_{0}(x, y) \in \mathcal{L}\left(E-P A^{\omega}\right)$ such that $E-P A^{\omega}+W K L+\Gamma \nvdash A$, where $\Gamma$ is the set of all true sentences having the form $\forall x^{\rho} F_{0}(x) \in$ $\mathcal{L}\left(E-P A^{\omega}\right)$ with $\operatorname{grad}(\rho) \leq 2$ and $F_{0}$ is quantifier-free ("True" here means valid in the full type structure of all set-theoretical functionals of finite type).

\section{Proof:}

$b-A C$ applied to $W K L^{\prime}$ yields $A: \equiv \exists \Phi \leq 1^{1(1)(1)} \forall f, g, x\left(\widehat{(\widehat{f})_{g}}((\overline{\Phi f g}) x)=0\right)$. One easily shows that $\Phi$ is not continuous w.r.t. $f$. Hence $E C F \mid \neq A$ (see Troelstra (1973),2.6.5 for the definition of $\left.E C F:=E C F\left(\omega^{\omega}\right)\right)$.

On the other hand $E C F \models E-P A^{\omega}+W K L+\Gamma$ : Since $E C F_{00}$ contains all functions $\omega^{\omega}, E C F \models$ $W K L$. Since the continuous $x^{\rho}$ are a subset of all set-theoretical $x^{\rho}(\operatorname{grad}(\rho) \leq 2 !)$, the truth of $\Gamma$ implies the truth of $[\Gamma]_{E C F} . \quad E C F \mid=E-P A^{\omega}$ follows from Troelstra (1973),2.6.5.

Our last theorem states that it is possible to extract bounds for (1) $\forall u^{1} ; v \leq_{1} t u \exists k^{0} D_{0}$-sentences which are proved from assumptions having the form (2) $\forall x^{0}\left(\forall w^{0} B_{0} \rightarrow \exists y \leq_{1} s x \forall z^{0} C_{0}\right)$ by analysing both the proof of $(2) \rightarrow(1)$ and a proof of the $\varepsilon$-version of (2). Thus, in contrast to assumptions $\forall x \exists y \leq s x \forall z A_{0}$, the truth of (2) is not sufficient for the extraction.

\subsection{Theorem}

1) Assume $(\rho=0 \wedge \tau$ arbitrary) or $(\rho=1 \wedge \tau=0)$ and

(i) $E-P A^{\omega}+A C^{\rho, \tau}-\mathrm{qf} \vdash \forall \alpha^{1} \exists \beta \leq_{1} r \alpha \forall n^{0} A_{0}(\alpha, \beta, n) \rightarrow$

$$
\forall x^{1}\left(\forall w^{0} B_{0}(x, w) \rightarrow \forall z^{0} \exists y \leq_{1} s x \bigwedge_{j=0}^{z} C_{0}(x, y, j)\right) \text { and }
$$

(ii) $E-P A^{\omega}+A C^{\rho, \tau}-\mathrm{qf} \vdash \forall x\left(\forall w^{0} B_{0}(x, w) \rightarrow \exists y \leq_{1} s x \forall z^{0} C_{0}(x, y, z)\right)$

$$
\left.\rightarrow \forall u^{1} ; v \leq_{1} t u \exists k^{0} D_{0}(u, v, k)\right) .
$$

Then from (i) one can extract a closed term $\chi \in T$ such that 
$(i)^{*} W E-H A^{\omega} \vdash \forall \alpha, n^{0} \exists \beta \leq r \alpha \bigwedge_{i=0}^{n} A_{0}(\alpha, \beta, i) \rightarrow$

$$
\forall x, z\left(\bigwedge_{i=0}^{\chi x z} B_{0}(x, i) \rightarrow \exists y \leq s x \bigwedge_{j=0}^{z} C_{0}(x, y, j)\right)
$$

¿From (ii)-using $\chi$-one can extract a closed $\Psi \in T$ such that

$(i i)^{*} W E-H A^{\omega} \vdash \forall \alpha, n \exists \beta \leq r \alpha \bigwedge_{i=0}^{n} A_{0}(\alpha, \beta, i) \rightarrow \forall u ; v \leq t u \bigvee_{k=0}^{\Psi u} D_{0}(u, v, k)$

2) An analogous result holds for $E \widehat{E-P} A^{\omega} \uparrow, \widehat{P R}$ and $W \widehat{E-} H A^{\omega} \uparrow$.

\section{Proof:}

1) ¿From (i) one concludes (using elimination of extensionality)

$W E-P A^{\omega}+A C^{\rho, \tau}-\mathrm{qf} \vdash \forall \alpha^{1} \exists \beta \leq_{1} r \alpha \forall n A_{0}(\alpha, \beta, n) \rightarrow \forall x^{1}, z^{0} \exists w^{0}\left(B_{0}(x, w) \rightarrow \exists y \leq_{1}\right.$ $\left.s x \bigwedge_{j=0}^{z} C_{0}\right)$.

By 3.5 there exists (eff.) a quantifier-free formula $F_{0} \in \mathcal{L}\left(W E-P A^{\omega}\right)$ such that

$$
W E-P A^{\omega} \vdash F_{0}(x, w, z) \leftrightarrow\left(B_{0}(x, w) \rightarrow \exists y \leq s x \bigwedge_{j=0}^{z} C_{0}\right)
$$

2.13.2 yields closed terms $\xi, \chi \in T$ such that

$$
W E-H A^{\omega} \vdash \forall x, z\left(\forall \alpha \exists \beta \leq \leq_{1} r \alpha \bigwedge_{i=0}^{\xi x z} A_{0} \rightarrow\left(\bigwedge_{i=0}^{\chi x z} B_{0}(x, i) \rightarrow \exists y \leq s x \bigwedge_{j=0}^{z} C_{0}\right)\right)
$$

(by 3.6, $b-A C-\forall^{b}$ is not needed to prove this conclusion),

which implies $(i)^{*}$.

(ii) implies (using again elimination of extensionality)

$W E-P A^{\omega}+A C^{\rho, \tau}-\mathrm{qf} \vdash \forall x \exists y \leq s x \forall z \exists w\left(\bigwedge_{i=0}^{w} B_{0}(x, i) \rightarrow \bigwedge_{j=0}^{z} C_{0}(x, y, j)\right) \rightarrow \forall u ; v \leq t u \exists k D_{0}$

Hence

$W E-P A^{\omega}+A C^{\rho, \tau}-\mathrm{qf} \vdash \exists Y \leq s, W^{001} \forall x, z\left(\bigwedge_{i=0}^{W x z} B_{0}(x, i) \rightarrow \bigwedge_{j=0}^{z} C_{0}(x, Y x, j)\right) \rightarrow \forall u ; v \leq t u \exists k D_{0}$. 
Therefore

$$
\begin{gathered}
W E-P A^{\omega}+A C^{\rho, \tau}-\mathrm{qf} \vdash \forall Y \leq s ; W, u ; v \leq t u \exists x, z, k\left(\left(\bigwedge_{i=0}^{W x z} B_{0}(x, i) \rightarrow \bigwedge_{j=0}^{z} C_{0}(x, Y x, j)\right)\right. \\
\left.\rightarrow \exists k D_{0}(u, v, k)\right) .
\end{gathered}
$$

Using functional interpretation (see Kohlenbach (1992),3.3) one can construct $\tilde{\Phi}, \tilde{\Psi} \in T$ from the given proof such that 


$$
\begin{aligned}
& W E-H A^{\omega} \vdash \forall Y \leq s ; W, u ; v \leq t u\left(\forall x\left(\bigwedge_{i=0}^{W x(\tilde{\Phi} Y W u v)} B_{0}(x, i) \rightarrow \bigwedge_{j=0}^{\tilde{\Phi} Y W u v} C_{0}(x, Y x, j)\right)\right. \\
&\left.\rightarrow \bigvee_{k=0}^{\tilde{\Psi} Y W u v} D_{0}(u, v, k)\right)
\end{aligned}
$$

(*) WE-HA $A^{\omega} \vdash \forall Y \leq s ; u ; v \leq t u\left(\forall x\left(\bigwedge_{i=0}^{\chi x(\tilde{\Phi} Y \chi u v)} B_{0}(x, i) \rightarrow \bigwedge_{j=0}^{\tilde{\Phi} Y \chi u v} C_{0}(x, Y x, j)\right)\right.$

$$
\left.\rightarrow \bigvee_{k=0}^{\tilde{\Psi} Y \chi u v} D_{0}(u, v, k)\right)
$$

By Kohlenbach (1992),2.15 one can construct $\tilde{\Phi}^{*}, \tilde{\Psi}^{*}, \chi^{*}, s^{*}, t^{*} \in T$ such that $W E-H A^{\omega} \vdash \tilde{\Phi}^{*} \operatorname{maj} \tilde{\Phi} \wedge \tilde{\Psi}^{*} \operatorname{maj} \tilde{\Psi} \wedge \chi^{*} \operatorname{maj} \chi \wedge s^{*} \operatorname{maj} s \wedge t^{*} \operatorname{maj} t$.

Define $\Phi:=\lambda u . \tilde{\Phi}^{*} s^{*} \chi^{*} u\left(t^{*} u\right), \Psi:=\lambda u . \tilde{\Psi}^{*} s^{*} \chi^{*} u\left(t^{*} u\right)$, then

$$
W E-H A^{\omega} \vdash \forall Y \leq s ; u ; v \leq t u\left(\Phi u \geq_{0} \tilde{\Phi} Y \chi u v \wedge \Psi u \geq_{0} \tilde{\Psi} Y \chi u v\right)
$$

Together with $(*)$ this implies

$$
\begin{aligned}
& W E-H A^{\omega} \vdash \forall Y \leq s ; u ; v \leq t u\left(\forall z \leq \Phi u ; x\left(\bigwedge_{i=0}^{\chi x z} B_{0}(x, i) \rightarrow \bigwedge_{j=0}^{z} C_{0}(x, Y x, j)\right)\right. \\
&\left.\rightarrow \bigvee_{k=0}^{\Psi u} D_{0}(u, v, k)\right) \\
& \Longrightarrow \\
& W E-H A^{\omega} \vdash \forall u\left(\exists Y \leq s \forall x ; z \leq \Phi u\left(\bigwedge_{i=0}^{\chi x z} B_{0}(x, i) \rightarrow \bigwedge_{j=0}^{z} C_{0}(x, Y x, j)\right)\right. \\
&\left.\rightarrow \forall v \leq t u \bigvee_{k=0}^{\Psi u} D_{0}(u, v, k)\right)
\end{aligned}
$$

$\stackrel{3.6}{\longrightarrow}$

$$
\begin{aligned}
(* *) W E-H A^{\omega} \vdash \forall u(\forall x \exists y \leq s x \forall z \leq & \Phi u\left(\bigwedge_{i=0}^{\chi x z} B_{0}(x, i) \rightarrow \bigwedge_{j=0}^{z} C_{0}(x, y, j)\right) \\
& \left.\rightarrow \forall v \leq t u \bigvee_{k=0}^{\Psi u} D_{0}(u, v, k)\right) .
\end{aligned}
$$

It remains to show 


$$
\begin{aligned}
& W E-H A^{\omega} \vdash \forall x, z\left(\bigwedge_{i=0}^{\chi x z} B_{0}(x, i) \rightarrow \exists y \leq s x \bigwedge_{j=0}^{z} C_{0}(x, y, j)\right) \rightarrow \\
& \forall u, x \exists y \leq_{1} s x \forall z \leq \leq_{0} \Phi u\left(\bigwedge_{i=0}^{\chi x z} B_{0}(x, i) \rightarrow \bigwedge_{j=0}^{z} C_{0}(x, y, j)\right)
\end{aligned}
$$

(Together with $(* *)$ and $(i)^{*}$ this implies $\left.(i i)^{*}\right)$.

Assume $(+) \forall x, z\left(\bigwedge_{i=0}^{\chi x z} B_{0}(x, i) \rightarrow \exists y \leq s x \bigwedge_{j=0}^{z} C_{0}(x, y, j)\right)$. Define primitive recursively in $x, u$ (in the sense of $T) z_{u, x}$ such that $z_{u, x}=\max \left\{z \leq_{0} \Phi u \mid \bigwedge_{i=0}^{\chi x z} B_{0}(x, i)\right\}$. Then $\bigwedge_{i=0}^{\chi x z_{u, x}} B_{0}(x, i)$.

By $(+)$ there exists an $y \leq s x$ with $(++) \bigwedge_{j=0}^{z_{u, x}} C_{0}(x, y, j)$. We show

$$
(+++) \forall z \leq_{0} \Phi u\left(\bigwedge_{i=0}^{\chi x z} B_{0}(x, i) \rightarrow \bigwedge_{j=0}^{z} C_{0}(x, y, j)\right):
$$

Case 1: $z \leq z_{u, x}$. Then by $(++) \bigwedge_{j=0}^{z} C_{0}(x, y, j)$.

Case 2: $\Phi u \geq z>z_{u, x}$. By the maximality of $z_{u, x}$ it follows that $\neg \bigwedge_{i=0}^{\chi x z} B_{0}(x, i)$ and hence $\bigwedge_{i=0}^{\chi x z} B_{0}(x, i) \rightarrow \bigwedge_{j=0}^{z} C_{0}(x, y, j)$

2) The proof is analogous.

\subsection{Remark}

1) The above theorem is usefull for the analysis of proofs which can be split into the parts

(i) $\forall \alpha \exists \beta \leq r \alpha \forall n A_{0} \rightarrow \forall x\left(\forall w B_{0} \rightarrow \exists y \leq s x \forall z C_{0}\right)$ and

(ii) $\forall x\left(\forall w B_{0} \rightarrow \exists y \leq s x \forall z C_{0}\right) \rightarrow \forall u ; v \leq t u \exists k D_{0}:$

One analyses separately the proof of (i), which is in particular a proof of

$$
(i)^{*} \forall \alpha \exists \beta \leq r \alpha \forall n A_{0} \rightarrow \forall x\left(\forall w B_{0} \rightarrow \forall z \exists y \leq s x \bigwedge_{j=0}^{z} C_{0}\right)
$$

and the proof of (ii) and combines the results to a bound for " $\exists k$ ".

In classical analysis there are interesting examples of proofs having this structure, e.g. the proof of the uniqueness of best Chebycheff approximation from de La Vallee Poussin (1919)/Natanson (1949), which we analyse in a subsequent paper using exactly the above strategy. 
2) Theorem 4.17 implies immediately (without 4.7 ) that $E-P A^{\omega}+A C^{\alpha, \beta}-\mathrm{qf}+W K L$ is conservative over $W E-H A^{\omega}$ w.r.t. $\forall u^{1} ; v \leq_{1} t u \exists k^{0} A_{0}$-sentences (analogous for the systems with restricted induction), since

$$
W \widehat{E-} H A^{\omega} \wedge \vdash W K L \leftrightarrow \forall f, g\left(T f \wedge \forall x(l \operatorname{th}(g x)=x \wedge f(g x)=0) \rightarrow \exists b \leq_{1} \lambda k .1 \forall x(f(\bar{b} x)=0)\right)
$$

and

$$
W \widehat{E-H} A^{\omega} \wedge \vdash \forall f, g\left(T f \wedge \forall x(l t h(g x)=x \wedge f(g x)=0) \rightarrow \forall x \exists b \leq \lambda k .1 \bigwedge_{j=0}^{x}(f(\bar{b} j)=0)\right) .
$$

[Correction (1993): Replace the condition ' $\left(\alpha=0 \wedge \beta\right.$ arbitrary) or $(\alpha=1 \wedge \beta=0)$ ' on $A C^{\alpha, \beta}-q f$ in $2.7,3.8,4.9 .2$ and 4.17 by ' $(\alpha=0 \wedge \beta \leq 1)$ or $(\alpha=1 \wedge \beta=0)^{\prime}$.]

\section{References}

\section{Bezem, M.A.}

Strongly majorizable functionals of finite type: a model for bar recursion containing discontinuous functionals. J. Symb. Logic 50, 652-600 (1985).

\section{Bridges, D.}

A constructive development of Chebycheff approximation theory. J. Approximation Theory 30, 99-120 (1980).

\section{Bridges, D.}

Lipschitz constants and moduli of continuity for the Chebyshev projection. Proc. Amer. Math. Soc. 85, no. 4, 557-561 (1982).

Clote, P.,Hajek, P.,Paris, J.

On some formalized conservation results in arithmetic. Arch. Math. Logic 30,201-218 (1990).

\section{De la Vallee Poussin, C.J.}

Lecons sur l'Approximation des Fonctions d'une Variable Reelle. Gauthier-Villars, Paris (1919).

\section{Feferman S.}

Theories of finite type. In: Barwise,J.(ed.) Handbook of mathematical logic (pp. 913-972). NorthHolland, Amsterdam 1977.

\section{Feferman S.}

Hilbert's program relativized: proof-theoretical and foundational reductions. J. Symb. Logic 53, 364-384 (1988).

\section{Friedman, H.}

Some systems of second order arithmetic and their use. Proc. of the 1974 International Congress of Mathematicians, Vancouver 1974, Vol. 1,235-242. Canadian Math. Congress (1975). 
Friedman, H.

Systems of second order arithmetic with restricted induction (abstract). J. Symb. Logic 41, 558-559 (1976).

Gdel, K.

Zur intuitionistischen Arithmetik und Zahlentheorie. Ergebnisse math. Kolloquium. Heft 4, 34-38 (1933).

Gdel, K.

ber eine bisher noch nicht benutzte Erweiterung des finiten Standpunktes. Dialectica 12, 280-287 (1958)

Hilbert, D.

ber das Unendliche. Math. Ann. 95, 161-190 (1926).

\section{Howard, W.A.}

Hereditarily majorizable functionals of finite type. In: Troelstra, A.S. (ed.) (1973), 454-461.

Kirby, L., Paris, J.B.

Initial segments of models of Peano's axioms. In: Lachlan, A.,Srebrny, M.,Zarach, A. (eds.), Set Theory and Hierarchy Theory V. Springer Lecture Notes in Math. Vol. 619, 211-226 (1977).

Kleene, S.C.

Introduction to Metamathematics. North Holland, Amsterdam 1952.

Kleene, S.C.

Recursive functionals and quantifiers of finite types I. Trans. AMS 91, 1-52 (1959).

\section{Kohlenbach, U.}

Theorie der majorisierbaren und stetigen Funktionale und ihre Anwendung bei der Extraktion von Schranken aus Beweisen: Effektive Eindeutigkeitsmodule bei besten Approximationen aus ineffektiven Eindeutigkeitsbeweisen. Dissertation, Frankfurt, pp. xxii+278 (1990).

\section{Kohlenbach, U.}

Pointwise hereditary majorization and some applications. Arch. Math. Logic. 31, pp. 227-241 (1992) (Misprints: p. 230 lines 22,34 replace $\lambda y^{\tau}(x) z^{*}$ by $\lambda y^{\tau} . z^{*}$, p. 231 lines 4,5 replace $\operatorname{maj}_{\rho_{i}}$ by $\underline{\operatorname{maj}}_{\rho_{i}}$ ).

\section{Kohlenbach, U.}

Remarks on Herbrand Analyses. Arch. Math. Logic. 31, pp. 305-317 (1992 A).

\section{Kreisel, G.}

Preface to Volume II of Reports of the Seminar on Foundations of Analysis, Stanford University (1963).

Kreisel, G.

Review of "Kleene/Vesley: Foundations of intuitionistic mathematics, especially in relation to recursive functions". J. Symb. Logic 31, 258-261 (1966). 
Kreisel, G., Mints, G.E., Simpson, S.G.

The use of abstract language in elementary metamathematics: Some pedagogic examples. In: Parikh, R. (ed.), Logic Colloquium (Boston 1972-73). Springer Lecture Notes in Mathematics Vol. 453, Berlin Heidelberg New-York (1975).

\section{Luckhardt, $\mathbf{H}$.}

Extensional Gdel functional interpretation. A consistency proof of classical analysis. Springer Lecture Notes in Mathematics Vol. 306, Berlin Heidelberg New-York (1973).

\section{Natanson, I.P.}

Konstruktive Funktionentheorie. Akademie-Verlag, Berlin (bersetzung des russischen Originals von 1949).

Scott, D.S.

Algebras of sets binumerable in complete extensions of arithmetic. In: Dekker, J.C.E. (ed.), Recursive function theory, Proceedings of Symposia in Pure Mathematics, vol. 5, AMS, Providence, R.I., 117121 (1962).

\section{Shoenfield, J.R.}

A relative consistency proof. J. Symb. Logic 19, 21-28 (1954).

Sieg, W.

Fragments of arithmetic. Annals of Pure and Applied Logic 28, 33-71 (1985).

Sieg, W.

Knig's lemma and fragments of arithmetic (abstract). J. Symb. Logic 52, 342-343 (1987).

Sieg, W.

Provable recursive functionals of theories with Knig's lemma. Rend. Sem. Mat. Univers. Politicn., Torino, 75-92 (1987 A).

Sieg, W.

Herbrand Analyses. Arch. Math. Logic 30, 409-441 (1991).

\section{Tait, W.W.}

Infinitely long terms of transfinite type. In: Crossley/ Dummett (eds.), Formal systems and recursive functions, 176-185, North-Holland, Amsterdam (1965).

\section{Troelstra, A.S.}

(Ed.) Metamathematical investigation of intuitionistic arithmetic and analysis. Springer Lecture Notes in Mathematics, Vol. 344, Berlin Heidelberg New-York (1973).

\section{Troelstra, A.S.}

Note on the fan theorem. J. Symb. Logic 39, 584-596 (1974).

Troelstra, A.S.

Some models for intuitionistic finite type arithmetic with fan functional. J. Symb. Logic 42, 194-202 (1977) 